To Appear in February 1997 A.J.

\title{
M-Subdwarfs: Spectroscopic Classification and the Metallicity Scalef
}

\author{
John E. Gizis \\ Palomar Observatory, 105-24, California Institute of Technology, Pasadena, California 91125, \\ e-mail: jeg@astro.caltech.edu
}

\begin{abstract}
We present a spectroscopic classification system for M-dwarfs and M-subdwarfs based on quantitative measures of $\mathrm{TiO}$ and $\mathrm{CaH}$ features in the region $\lambda \lambda 6200-7400 \AA$. Our sample of cool stars covers the range from solar metallicity stars to the most extreme subdwarfs known. Using synthetic spectra computed by Allard and Hauschildt (1995), we derive metallicities for the stars. Stars are classified as dwarfs (M V), subdwarfs (sdM), or extreme subdwarfs (esdM). These classifications correspond to $[m / H] \approx 0.0,-1.2$, and -2.0 respectively. Our metallicity scale agrees with theoretical HR diagrams and HST globular cluster measurements. We discuss some nearby subdwarfs of particular interest in light of our metallicity scale.
\end{abstract}

\section{Introduction}

The vast majority of stars are M-dwarfs, main sequence stars whose spectra are dominated by molecular absorption. They have lifetimes much greater than the age of the universe which makes them an important fossil record of Galactic history. Their potential is largely unrealized because investigations of their properties have been hampered by the complex absorption spectra of diatomic and triatomic molecules. In particular, theoretical model atmospheres face serious difficulties; as a result, traditional methods of determining abundances from high resolution spectra of weak atomic lines are inapplicable, requiring the use of other techniques.

A fundamental astronomical tool is a classification system that spans the range of observed properties, allowing an estimate of the effective temperature $\left(T_{\text {eff }}\right)$, luminosity, and abundance $([m / H])$ from a spectrum by comparison to standard stars with known properties. The system also should also allow identification of rare objects with unusual properties. The MKK dwarf spectral sequence (Morgan et al. 1943) extended to M2 V and only included Population I objects. As progressively cooler stars have been discovered the classification system has been extended to M6.5 by Boeshaar (1976) and M9 (Kirkpatrick et al.1991, hereafter KHM; see also Bessell 1991).

\footnotetext{
${ }^{1}$ Observations were made partially at the 60 -inch telescope at Palomar Mountain which is jointly owned by the California Institute of Technology and the Carnegie Institution of Washington
} 
The KHM system uses features in the wavelength ranges $\lambda \lambda 6950-7500 \AA$ and $\lambda \lambda 8400-8950 \AA$ observed at $\sim 18 \AA$ resolution or better, and Henry et al. (1994, hereafter HKS) have applied it to cool stars within 8 parsecs. A slightly different approach was taken by Reid et al. (1995, hereafter RHG) and Hawley et al. (1996, hereafter HGR) who used measurements of the 7100 $\mathrm{TiO}$ bandhead at higher resolution $(\sim 3 \AA)$ to classify most of the known M-dwarfs within 25 parsecs. The standards from KHM and HKS were used to place the RHG observations on the standard system.

The spectral classification system is thus now well-defined for the near solar abundance Mdwarfs of the Galactic Disk. The situation for low metallicity stars, traditionally called subdwarfs, is much more confused. Although many spectra of individual stars have been published, there is no consistent M-subdwarf classification system. Examples of metal-poor M-subdwarfs were spectroscopically identified as long ago as Joy (1947), although "later investigators had difficulty in recognizing his criteria" (Oort 1965). Mould and McElroy (1978) discussed "old disk subdwarfs" which were less metal poor than other subdwarfs on the basis of $\mathrm{TiO}$ and $\mathrm{CaH}$ indices. Ake and Greenstein (1980), following a spectroscopic survey of high velocity stars, published spectra of four "extreme subdwarf M stars" which appeared to have "extreme metal deficiency" compared to the usually recognized M-subdwarfs. Similar stars were identified spectroscopically in a search for nearby white dwarfs (Liebert et al. 1979), a search for Population II halo stars (Hartwick et al. 1984), and a survey of cool M-dwarfs (Bessell 1982), all targeted at faint, high-proper motions stars. Recently, trigonometric parallaxes of 17 extreme subdwarfs have been measured, confirming their subluminosity (Monet et al. 1992, hereafter M92). Thus objects selected by different criteria can be called subdwarfs by different authors. M92 also found that there is a gap between their extreme subdwarf sequence and the "less extreme" subdwarf and disk sequence in the $M_{V}$ vs. V-I HR diagram - their preliminary interpretation was that this gap represented a real lack of "intermediate" metallicity stars. In any case, the physical properties of the various subdwarf types remain in doubt; for example, M92 argue that their extreme subdwarfs have $[\mathrm{m} / H] \sim-1.7$ whereas Eggen (1996) suggests they have $[m / H] \sim-2.5$ to -3.5 .

Here we present a self-consistent set of spectroscopic observations of cool metal-poor stars drawn from a variety of sources. Selection of our subdwarf candidates and the data reduction procedures are discussed in Section 2. In Section 3, we discuss empirical molecular bandstrengths and suggest a two-dimensional classification system. In Section 4 , we compare our spectra to model atmospheres and deduce metallicities. In Section 5, we discuss the use of color-color and HR diagrams. Some notable individual stars are discussed in Section 6. The results are summarized in Section 7 .

\section{Observations}




\subsection{Sample Selection}

Identification of a metal-poor or halo population can be difficult and ambiguous. It is well known from studies of hotter (F, G, and $\mathrm{K}$ ) stars that there is no one-to-one correspondence between kinematics and metallicity because there is overlap between the properties of the disk and halo components (e.g., Mihalas and Binney 1981). Even among local stars with $v_{\text {tan }}>100 \mathrm{~km} \mathrm{~s}^{-1}$, the halo (Population II) is outnumbered approximately ten to one (Schmidt $1975)$ by high velocity, slightly metal-poor disk stars (the Intermediate Population II, reviewed by Majewski 1993). Despite the name Intermediate Population II (IPII), these stars have a mean metallicity $([m / H] \sim-0.6)$ close to that of the disk $([m / H] \sim 0.0)$, although the exact abundance distribution of the IPII is difficult to determine (Carney et al. 1989). In contrast, a conservatively selected field halo sample (Laird et al. 1988) (restricted to stars that have $v_{\text {tan }}>220 \mathrm{~km} \mathrm{~s}^{-1}$ or $V<-220 \mathrm{~km} \mathrm{~s}^{-1}$ ]) has a distribution in $[\mathrm{m} / H]$ that peaks at -1.7 with FWHM $\sim 1.2$ dex, and includes tails that extend down to very low abundances $(8 \%$ of the stars have $[\mathrm{m} / H]<-2.5)$ and up to IPII-like abundances $(9 \%$ have $[\mathrm{m} / H]>-1.0)$. We therefore a priori expect that kinematically selected samples with loose selection criteria (e.g., $v_{\tan }>100 \mathrm{~km} \mathrm{~s}^{-1}$ ) will include many IPII stars that have $[m / H]>-1$, and we also expect to see a large range of metallicities even among "true" halo stars.

We have chosen our objects from a number of sources; however, virtually all have been identified in proper motion surveys and appear in the Luyten LHS catalog (Luyten 1979) and most are in the the Lowell Proper Motion Survey (Giclas et al. 1971). These surveys give proper motions, photographic magnitudes, and low-precision color indices which are by themselves not adequate to isolate a halo or metal-poor sample. However, many late-type candidate subdwarfs have been identified in followup surveys. Since we use a variety of sources, selection criteria are ill-defined but typically depend on the star's velocity, color, or spectral features. We particularly favor stars with measured trigonometric parallaxes. These effects are not important for this work since we are not setting out to measure statistical quantities. Of particular note are the Schmidt (1975) complete sample of stars with $\mu>1.295^{\prime \prime} \mathrm{yr}^{-1}$ and $m_{p g}<15.95$, the Greenstein (1989) sample of cool halo stars, and the M92 and Ruiz and Anguita (1993, hereafter RA) samples of CCD parallax stars. Other objects have been chosen from lists of unusual stars in photometric followups (Dawson and Forbes 1989, Dawson and Forbes 1992, Reid 1982, Leggett 1992) Finally, it should noted that the Giclas catalog only goes to $m_{p g} \sim 17$ and is incomplete for $m_{p g} \geq 16$. Schmidt (1975) showed that the apparent lack of $M_{p g}>13.5\left(M_{V} \sim 12\right)$ is due to this apparent magnitude limit. The Luyten searches reach $m_{R} \sim 20$ but are incomplete both for $m_{R}>18$ and $\mu>2.5^{\prime \prime} / \mathrm{yr}$ (Dawson 1986). The latter limit implies that stars with typical halo tangential velocities of 220 and $300 \mathrm{~km} \mathrm{~s}^{-1}$ would not have been detected within 18 and 25 parsecs respectively.

In addition to the candidate subdwarfs selected above, we have utilized the RHG observations

\footnotetext{
${ }^{2}$ We use the standard notation of $(\mathrm{U}, \mathrm{V}, \mathrm{W})$ for the space velocity components; note that $\mathrm{U}$ is positive towards the Galactic Center $(l=0, b=0)$.
} 
of 1700 M-dwarfs from the preliminary Third Catalog of Nearby Stars (Gliese and Jahreiss 1991, CNS3) as a reference solar abundance sample. We note particular use of two subsets from this sample. First, we use the stars within eight parsecs, selected to be either single or well separated from their companions, which outline the detailed structure in the HR diagram (e.g., Gizis and Reid 1996). Distances were adopted from RHG, but the stars were required to have accurate trigonometric parallaxes with Lutz-Kelker (1973) corrections less than 0.1 magnitudes. Second, we also use the nearby stars with $v_{\text {tan }}>100 \mathrm{~km} \mathrm{~s}^{-1}$.

\subsection{Spectroscopic Observations and Data Reduction}

Spectra were obtained at the Palomar 60 in. telescope, the Hale 200 in. telescope, and the Las Campanas Du Pont 100 in. telescope. We used the G-mode of the Palomar 60 in. spectrograph (McCarthy 1985), a 1 arcsecond slit, and a $600 \mathrm{l} / \mathrm{mm}$ grating blazed at $6500 \AA$, yielding $1.5 \AA \mathrm{pix}^{-1}$. Candidate subdwarfs were observed in May 1994, September 1994, and January 1996 while some CNS3 stars were observed with the same setup in 1993 and 1994 (RHG). At the Hale 200 in. telescope we used the double spectrograph. In August 1995 with the blue camera was set to observe $6000-6900 \AA$ and the red camera was set to $6700-8000 \AA$ using 600 l/mm gratings blazed at $4000 \AA$ and $10000 \AA$ respectively. In October 1995 , a new red camera was installed in the double spectrograph, which we used in all subsequent runs to observe the region $\lambda 6000-7400 \AA$ at $1.4 \AA \mathrm{pix}^{-1}$. The 100 in. telescope observations used the modular spectrograph with a 1200 line grating blazed at $7500 \AA$. The resolution at all telescopes was $3-4 \AA$. At all telescopes, neon and argon arcs were taken after each observation in order to eliminate the effects of instrument flexure. The data were extracted, sky subtracted, and wavelength calibrated using the FIGARO package. Flux standards (Gunn and Oke 1983 and Baldwin and Stone 1984) were used to set the data on an $F_{\nu}$ scale. Since observing conditions were usually non-photometric with seeing worse than 1 arcsecond, the fluxes are not absolute.

Radial velocities were measured using the FIGARO cross correlation task SCROSS and corrected to a heliocentric frame using VHELIO. M-dwarf radial velocity standards were drawn from Marcy and Benitz (1986). For the Hale 200 in. telescope observations, the extreme subdwarf LHS 1174 was also used as a cross correlation standard. A radial velocity of $-112 \pm 2 \mathrm{~km} \mathrm{~s}^{-1}$ was adopted based upon a Keck HIRES spectrum (Reid, personal communication). Using either the extreme subdwarf or disk M-dwarfs did not make a significant change in the derived velocities, which have an uncertainty of $\pm \sim 20 \mathrm{~km} \mathrm{~s}^{-1}$. We have 14 stars in common with the more precise measurements of Dawson and De Robertis (1988, 1989) - we find a mean difference of $10 \mathrm{~km} \mathrm{~s}^{-1}$ and a standard deviation of $26 \mathrm{~km} \mathrm{~s}^{-1}$. The three stars with differences of more than $30 \mathrm{~km} \mathrm{~s}^{-1}$ are LHS 161, 205a, and 479. 


\section{Bandstrengths and a Subdwarf Spectroscopic Sequence}

Molecular features are typically very broad (tens or hundreds of Angstroms) and often are asymmetric. In M-dwarfs, there are few points that are relatively clear of absorption, but model atmospheres (Allard and Hauschildt 1995) show that even these are depressed from the "true" continuum. Since absolute measurements of the strength of features are therefore impossible, we instead measure molecular features with bandstrength indices defined as

$$
R_{\text {ind }}=\frac{F_{W}}{F_{\text {cont }}}
$$

where pseudo-continuum $\left(F_{\text {cont }}\right)$ region and the feature $\left(F_{W}\right)$ wavelength limits are taken from RHG and listed here in Table 0. In the case of the CaH1 index, two sidebands are used to estimate the pseudo-continuum flux $\left(S_{1}\right.$ and $\left.S_{2}\right)$. As in RHG, the spectral indices have an accuracy of $\pm 0.02-0.04$. Note that our experience with these indices show that all except $\mathrm{CaH} 2$, which uses widely separated pseudo-continuum and feature regions, are insensitive to the flux calibration errors. CaH2 shows systematic offsets of up to 0.03 from observing run to observing run. The measured indices for our stars are given in Table 2.

The KHM M-dwarf spectral types provide an excellent shorthand description of the spectral properties of Galactic Disk stars - we find that over the range K7 - M6 the relations

$$
\begin{gathered}
S p=-9.64 \times \mathrm{TiO} 5+7.76 \\
S p=7.91 \times \mathrm{CaH}^{2}-20.63 \times \mathrm{CaH} 2+10.71 \\
S p=-18.00 \times \mathrm{CaH} 3+15.80
\end{gathered}
$$

are accurate to \pm 0.5 subclass, where $\mathrm{K} 5$ and $\mathrm{K} 7$ are $S p=-2$ and -1 respectively. As described by RHG, the CaH1 index saturates at $\sim M 3$, beyond which it does not show much temperature dependence. Although TiO5 was used by RHG to classify the stars, the two CaH relations are equally good spectral type indicators for near solar metallicity stars.

Figure 11 shows the three $\mathrm{CaH}$ indices plotted against $\mathrm{TiO} 5$ for our candidate metal-poor stars and the eight parsec disk sample. [3 Fifty stars lie significantly below the mean $\mathrm{CaH}$ vs TiO5 relations, a significant difference compared with only 5 of 1685 stars in RHG. There appears to be a fairly well defined sequence to the lower right of each figure - in Figure 1 $1 \mathrm{~b}$ a high-order polynomial illustrates the cutoff adopted. We will call the stars below this cutoff the extreme M-subdwarfs. Note however that the region between the extreme M-subdwarf sequence and the disk M-dwarfs is well represented in our sample.

The extreme subdwarf sequence, with the notable exception of LHS 453, has very weak but detectable $\mathrm{TiO}$ absorption at the resolution of our observations. However, even the coolest star,

\footnotetext{
${ }^{3}$ The relations defined by all of the M-dwarfs in the northern CNS3 are plotted in RHG's Figure 4.
} 
LHS 1742a $\left(M_{V}=14.43\right)$, has only the TiO strength of a disk M1 star - the rest of the sequence is equivalent to K7 or K5 stars according to equation 1. Indeed, LHS 1174 was classified "sdK7-M1" by Liebert (1991, personal communication reported in M92). A TiO classification thus compresses the entire sequence of M-subdwarfs into only three subclasses even though Figure 1 shows there is as much or more variation in the $\mathrm{CaH}$ strengths as $\mathrm{TiO}$ variation in the disk M-dwarfs. Use of equations 2 or 3 gives spectral types of M5.5 (CaH2) or M8 (CaH3, extrapolated) for LHS 1742a.

In many circumstances a simplified shorthand notation may be more useful than measurements of specific molecular features, even though Figure 1 shows that there is a full two-dimensional continuum in the properties of M-subdwarfs. Using the $\mathrm{TiO}$ and $\mathrm{CaH}$ indices as a guide, a sequence of low abundance M spectral standards can be defined. While this is the first subdwarf standard sequence to be defined, historical precedent supports using the prefix "sd" before the M spectral class. (Luminosity class VI should not be used for subdwarfs according to Jaschek and Jaschek 1987.) In the past, LHS 64 has been classified sdM1 (Joy 1947), very similar to the "sdK7-M1" classification for LHS 1174. Unfortunately the use of "sd" alone throws away much information - although both types of subdwarfs above are significantly different from Population I stars, they also differ at least as much from each other, as seen in Table 2 and Figure 1. Second, as discussed above, use of $\mathrm{TiO}$ alone would artificially suppress much of the variance among the extreme subdwarfs.

We therefore propose the following classification system, which consists of both a prefix (M, sdM, esdM) and a numerical subclass. The classification system works as follows. First, determine if the star if a subdwarf of some kind using CaH1. Second, use CaH2 to determine if the star is a sdM or esdM. Finally, assign a numerical subclass. Each step is described in detail below.

In the first step, stars that show significantly stronger $\mathrm{CaH}$ absorption for their TiO5 strength compared with disk dwarfs are designed subdwarfs with the prefix "sdM" or "esdM". Quantitatively, we define significant as lying 0.07 below the mean Population I CaH1-TiO5 relation of the RHG stars. If the other indices are unavailable, 0.06 below the RHG CaH2-TiO5 or CaH3-TiO5 relations is equivalent. For the coolest stars ( $\mathrm{TiO} 5<0.49)$, CaH2 or CaH3 must be used since the disk CaH1 relation is "saturated." The equations for these cutoffs are:

$$
\begin{aligned}
& \mathrm{CaH} 1<0.695 \times \mathrm{TiO}^{3}-0.818 \times \mathrm{TiO}^{2}+0.413 \times \mathrm{TiO} 5+0.651 \\
& \mathrm{CaH} 2<0.968 \times \mathrm{TiO}^{3}-1.358 \times \mathrm{TiO}^{2}+1.315 \times \mathrm{TiO} 5-0.033 \\
& \mathrm{CaH} 3<0.639 \times \mathrm{TiO}^{3}-1.199 \times \mathrm{TiO}^{2}+1.161 \times \mathrm{TiO} 5+0.307
\end{aligned}
$$

Stars that fulfill Equations 4, 5, or 6 are either sdM or esdM. Equation 4 is plotted in Figure 1 1a. In the second step, the extreme subdwarfs are identified. A high order polynomial, shown in Figure 1 $1 \mathrm{~b}$, was used to interpolate between arbitrarily chosen points to define a cutoff in $\mathrm{CaH} 2$ vs. TiO5. The coordinates of the points, expressed as (TiO5,CaH2), are $(0.0,-0.1),(0.433,0.234),(0.600,0.303),(0.800,0.456),(0.905,0.626),(0.977,0.788)$, and $(1.020,0.914)$. The sdM lie above these points and the esdM lie below. The separation between the subdwarfs 
and extreme subdwarfs is natural (at least for this sample) for the M stars, but at the hotter end the weakness of the features makes the sequences merge. At the cool end, the relation is not defined beyond $\mathrm{TiO} 5 \sim 0.6$, but the points given prevent the interpolating polynomial from inconveniently crossing the disk sequence.

Finally, the numerical subclass is determined. The definition of the numerical subclasses is problematic. Larger numbers correspond to lower temperatures, but since effective temperatures are not well determined for cool stars it is impossible to ensure that sdM subclasses correspond to the same temperatures as M V stars or esdM stars. Some authors (e.g., Hamilton and Stauffer 1993) have used pseudocontinuum indices to assign types for late-type disk M-dwarfs, but we find that the pseudocontinuum points available in our spectral range show more scatter than the bandstrength indices we are using. We adopt Equation 2 as the definition of numerical subclasses for the sdM and esdM because this feature shows the same range $(0.2 \lesssim \mathrm{CaH} 2 \lesssim 1.0)$ for all metallicities. Use of the disk relation for the CaH3 feature (Equation 3) or the TiO5 feature (Equation 1) leads to very large and very small ranges in the spectral type for the esdM. The subclass, however, is relatively uncertain when using only the CaH2 feature since the spectral type determination then depends upon only a single feature. We have therefore determined the following linear fits:

$$
\begin{aligned}
& S p_{\mathrm{sdM}}=-16.02 \times \mathrm{CaH} 3+13.78 \\
& S p_{\mathrm{esdM}}=-13.47 \times \mathrm{CaH} 3+11.50
\end{aligned}
$$

The numerical subclasses listed in Table 2 are the average of the CaH2 relation (Equation 2) and the appropriate CaH3 relation (Equation 3, 7, or 8). We continue to classify stars as "sd" or "esd" as early as K7. For earlier stars, we assign classes of only sdK (or sdG for stars with strong $H \alpha$ absorption). The cooler sdK can be distinguished in Figure 1 where they have stronger CaH1 relative to the $\mathrm{K}$ stars presented in RHG. These features are quite weak so the measurement errors are more important. The classifications of the $\mathrm{K}$ stars are therefore not as certain and should be regarded with caution - they are presented here to show the continuity with the M-subdwarfs. The new classifications are listed in Table 2. Figures 2 and 3 show sequences of spectra for the subdwarfs and extreme subdwarfs respectively.

The cool ends of the sequences are not yet well-defined, since there are as yet observations of only a few very cool spectroscopic subdwarfs. LHS 407 has been classified as sdM5.0. Although in Figure 1 it is well to the left of the esdM, it lies with the extreme subdwarfs in Figure 1 and the HR diagram (Figure 7). It is easily distinguished from LHS 1742a (esdM5.5), which is at present the coolest extreme subdwarf known. LHS 3061 is similar enough to LHS 1742a to also be esdM5.5, but it is slightly hotter according to its spectral indices and M92 V-I color $\left(\Delta_{V-I}=0.13\right)$. LHS 3409, observed by RHG, is apparently more metal-rich than LHS 407, yet its indices are significantly different from the Population I, or even most stars with velocities typical of the IPII. We have therefore called it sdM4.5. LHS 3480 is a similar star. A considerably redder sdM is the star LHS 377 (shown by M92 to have $M_{V}=15.66$ ). Its TiO5 index is equivalent to an M5.5 V star, but CaH2 gives the classification sdM7 (M92 report that Boeshaar and Liebert 
call the star sdM5 in a private communication). Finally, we have also observed TVLM 832-42500 which is subluminous in the I-K vs $M_{K}$ diagram, which suggests it has $[m / H] \sim-0.5$ to -1.0 (Tinney et al. 1995). It is at spectral type $\sim$ M7 V where the indices break down; however, compared to the RHG stars of similar TiO5 strength it has slightly stronger $\mathrm{CaH} 2$ and $\mathrm{CaH} 3$, suggesting it is indeed slightly more metal poor, or at least spectroscopically different. Its TiO5 and CaH2 absorption is slightly stronger than LHS 377 but the CaH3 absorption is much weaker. It thus appears to be more metal rich than LHS 377. A proper understanding of the distinction between cool sdM vs. esdM will require identification of more very cool stars from the LHS catalog. However, since we determine the $\mathrm{M}$ spectral subclass by the quantitative $\mathrm{CaH} 2 \mathrm{index}$, the classification of M4.5 and M5.5 for the stars above is well-defined in our system.

The relative strengths of bandstrength indices contains more information than just a simple classification. HGR show that there are systematic differences in the $\mathrm{TiO}$ indices of $\mathrm{dMe}$ and dM stars. In Figure 4 , we plot the ratio $\mathrm{CaH} 3 / \mathrm{CaH} 2$ as a function of $\mathrm{CaH} 2$ strength for all the RHG stars and for the sdM and esdM. The sdM tend to lie below the Population I sequence. The extreme M-subdwarfs lie systematically well below the sequence. The trend is that the first band of $\mathrm{CaH}(\mathrm{CaH} 3)$ is stronger relative to the entire bandhead $(\mathrm{CaH} 2)$ - similar to the behavior seen in TiO for late type M-dwarfs. Since the deeper band must reflect conditions higher in the atmosphere, the difference might be caused by a slight surface gravity difference and/or a lower temperature in the CaH3 formation region compared to sdM and dM. Detailed modeling is necessary to understand this difference.

\section{Model Atmosphere Fitting}

Interpretation of the observed spectra in terms of the physical parameters effective temperature $\left(T_{e f f}\right)$ and metallicity $([\mathrm{m} / H])$ requires the use of model atmospheres. In principle,

surface gravity is also a relevant parameter; however, both theory and observations indicate dwarf stars have $\log g \sim 5.0 \pm 0.2$ (Leggett et al. 1996) and we therefore restrict analysis to $\log g=5.0$ models. We use the Extended Model grid computed by Allard and Hauschildt (1995, hereafter $\mathrm{AH})$ which assumes local thermodynamic equilibrium (LTE).

The theoretical uncertainties and limitations of the atmosphere models have been extensively discussed by AH. Although there are uncertainties in the appropriate treatment of the physics such as non-LTE effects, the treatment of molecular opacities (usually the Just Overlapping Line Approximation is used in these models), and the treatment of convection, the primary limitation is simply the lack of both laboratory data and theoretical computations of the electronic band oscillator strengths. In particular, no data exist for the $\mathrm{CaH}$ molecule which is extremely important in our spectral region. In Figure 5 , we show a synthetic spectrum based upon a solar metallicity model and a representative disk star, the KHM M 4.0 V standard Gl 402. (The treatment of the $\mathrm{TiO}$ molecule in solar metallicity models has recently been improved by Allard and Hauschildt in the "NextGen" model). The spectra are in good general agreement, although the specific strengths 
of the molecular features are incorrect, as expected. In particular, the $\mathrm{CaH}$ feature at $\sim 6950 \AA$ is too strong in all models compared to the observed spectra. However, AH show that their models capture the general overall behavior of metal-poor stars - with decreasing metallicity the hydride bands and atomic lines both increase in strength - the latter due to an increase in gas pressure.

Figures 2 and 3 clearly show the expected increase in the atomic line strength with increasing hydride strength. Until accurate data or calculations are available for all the important molecules, but especially the hydride bands, any comparison between observations and theory will remain uncertain. Since such information is not likely to be available for many years, the uncertainties of this study will not reduced in the near future. Other authors have compared the AH models to cool solar abundance and metal-poor dwarfs using broad band colors and low-resolution optical and infrared spectra (Dahn et al. 1995; Leggett et al. 1996) or higher resolution infrared spectra (Jones et al. 1996). Our study is most sensitive to variations in $\mathrm{CaH}$ and $\mathrm{TiO}$ which dominate our more limited wavelength region. It should also be noted that the metal poor models are computed assuming scaled solar abundances. If $[O / C]$ is enhanced in the Population II M-subdwarfs, as it is in hotter Population II stars, it will affect the strength of $\mathrm{TiO}$ and $\mathrm{H}_{2} \mathrm{O}$ absorption (Mould 1976).

Due to the uncertainties in the molecular data, the $\mathrm{AH}$ models cannot be used to predict the bandstrengths measured in Section 3, nor are they able to reproduce the CaH3/CaH2 relation in Figure 1 . Instead, we compare the spectral region $(\lambda \lambda 6200-7300)$ by minimizing the least squares difference $\left(\chi^{2}\right)$ between the observed spectrum and the models. We first shift the observations to rest velocity and vacuum wavelengths, convert to $F_{\lambda}$, and rebin to $2 \AA$ steps. The models are convolved with a $\sigma=2 \AA$ Gaussian to approximate the instrumental resolution (in practice, this has negligible effects since the synthetic spectra often show $50 \AA$ wide "features" due to the computation technique). The extended models have been computed for steps of 0.5 dex in metallicity between $[\mathrm{m} / H]=0.0$ and $[\mathrm{m} / \mathrm{H}]=-4.0$. We consider $100 \mathrm{~K}$ steps for the temperature range $2500 K \leq T_{\text {eff }} \leq 4000 K$ (higher temperatures are not available and lower temperatures are irrelevant to the stars under consideration here). For temperatures that were not calculated $\left(T_{\text {eff }}\right.$ $=2600,3100,3400,3600,3800$, and $3900 \mathrm{~K}$ ), we approximate the synthetic spectra by linearly interpolating between the two nearest temperatures.

The best fit model parameters for the subdwarf candidates are listed in Table 3. We have also fit the eight parsec sample of single disk M-dwarfs; below $3800 \mathrm{~K}$, the spectra are best matched by solar metallicity models, as expected. Above $3800 \mathrm{~K}$, the fits are poor because the molecular features are too weak to constrain the fits well. We therefore report only those fits with $T_{\text {eff }}<3800 \mathrm{~K}$. The uncertainties from the fitting procedure are the grid spacing of $\pm 0.5 \mathrm{in}[\mathrm{m} / \mathrm{H}]$ and $\pm 100 \mathrm{~K}$ in $T_{\text {eff }}$. The systematic uncertainties are unknown, but are dominated by the errors in the synthetic spectra discussed above. We note that different temperature scales for the disk M-dwarfs have historically had systematic differences of up to $300 \mathrm{~K}$ (e.g., Leggett et al. 1996) and our fits are subject to all the same uncertainties. Compared to Leggett et al. (1996), our fits give somewhat higher temperatures $\left(\Delta T_{\text {eff }}=150 \mathrm{~K}\right.$ for LHS $377, \Delta T_{\text {eff }}=400 \mathrm{~K}$ for LHS 57). 
The extreme subdwarfs have best fits at $[m / H] \sim-2$. Figure 6 shows one representative fit.. The coolest extreme subdwarf, LHS 1742a, has an effective temperature of $3300 \mathrm{~K}$. A difference of one extreme subdwarf spectral subclass derived from CaH2 corresponds to an approximately 100

$\mathrm{K}$ difference in temperature. Some authors (e.g., Eggen 1996) have argued that the the extreme subdwarfs are likely to have $[m / H]=-2.5$ to -3.0 , whereas our least-squares fitting of model atmospheres gives metallicities a factor of 10 higher. The model atmospheres show that the most obvious qualitative difference is that $\sim 7100 \AA$ TiO features for the -2.5 and -3.0 models should be completely absent. The observed spectra, however, show that although the feature is weak it does exist in our extreme subdwarfs (except LHS 453), supporting the model atmosphere fits. LHS 453 completely lacks the TiO5 feature (the $\mathrm{TiO}$ feature at $6200 \AA$ is also missing) and therefore is more metal poor than the other esdM. The best fit is $[m / H]=-2.5$. For higher metallicities $([m / H]=-1.0)$ the $\mathrm{TiO}$ feature remains strong which is qualitatively seen in the stars classified sdM. Indeed, the sdM have best $[\mathrm{m} / H]$ fits of -1.0 to -1.5 . For the stars that were not classified subdwarfs, the model fits give $[\mathrm{m} / \mathrm{H}]$ of 0.0 to -1.0 .

In summary, the spectroscopic classification system of Section 3 can be used to derive metallicities based upon our fits to AH synthetic spectra. Stars classified as extreme M-subdwarfs (esdM) have $[m / H]$ of $-2.0 \pm-0.5$, whereas sdM have a metallicity of $-1.2 \pm 0.3$. Stars that do not show significant differences from the RHG disk sample in their $\mathrm{CaH}$ indices have $[m / H]>-1.0$. When model atmospheres are able to accurately reproduce the $\mathrm{CaH}$ and $\mathrm{TiO}$ bandheads more accurate metallicities should be possible.

\section{HR diagrams}

HR diagrams are an important tool for the study of cool stars. Observational HR diagrams are a critical test of stellar structure theory, provided that the transformation between theoretical temperatures and luminosities and observed quantities (colors and absolute magnitudes) can be made. In practice there has been disagreement over the Population I temperature scale (Leggett et al. 1996) and relatively little discussion of the temperature of Population II subdwarfs. The HR diagram can also be used to predict absolute magnitude from observed color and thus is used in interpretation of star count data and derivation of the luminosity function. Finally, derivation of the mass function from the luminosity function of metal-poor stars depends upon the quality of the theoretical mass-luminosity relations and evolutionary tracks since there are no cool Population II binaries with mass determinations.

Table 1 lists optical photometry and trigonometric parallaxes from the literature for the stars in our sample. We have adopted the Cousins R and I system and where necessary have used the color transformations compiled by Leggett (1992). Because the photometry is from heterogeneous sources of varying quality, in some cases the colors may be incorrect by as much as 0.1 magnitudes but the typical accuracy should be about 5\% (Leggett 1992). In addition, the transformations have been derived for near solar metallicity stars and may be inaccurate for subdwarfs. Absolute 
parallaxes were taken from the preliminary Yale Parallax Catalog (van Altena et al.1991) when available. In the case of the CCD relative parallaxes reported by RA, we added a correction of 0.5 milliarcseconds to the parallaxes to transform them to absolute measurements. This approximately matches the correction applied by M92 for similar magnitude stars and the uncertainty in this correction is much less than the uncertainties in the relative parallaxes. Although the measured parallax is the best estimator of the distance to any particular star, there is a statistical bias to underestimate the distance and hence underestimate the the mean luminosity of the sample stars. Lutz and Kelker (1973) derived statistical corrections to the observed absolute magnitudes as a function of $\sigma / \pi$ and showed that only parallaxes with $\sigma / \pi<0.2$ are useful. However, selecting the stars by proper motion reduces the statistical correction (Hansen 1979). Since the selection of our sample is not well-defined, we do not apply any statistical correction to the absolute magnitudes, but we list the Lutz-Kelker corrections (derived from the approximation given by Hansen 1979 and defined as $\left.M_{\text {true }}=M_{o b s}+\Delta_{L K}\right)$ as a guide to likely biases in the sample. The V-I, $M_{V}$ diagram is shown in Figure 7. The eight parsec single stars are plotted with Lutz-Kelker corrections applied, but the sample is restricted to stars with corrections less than 0.1 magnitude. We also plot the B-V version of this diagram in Figure 8, including the bluer $\mathrm{K}$ subdwarfs in the sample and the disk $\mathrm{G}$ and $\mathrm{K}$ dwarfs within eight parsecs from the CNS3. It is well known that B-V colors are poorly suited for estimating absolute magnitudes for the disk M-dwarfs (in the disk sequence $M_{V}$ changes by 4 magnitudes in the range $1.4<\mathrm{B}-\mathrm{V}<1.6$.) In our diagram, the sdM and esdM sequences cross the disk sequence at $\mathrm{B}-\mathrm{V} \sim 1.5$, and the reddest sdM and esdM actually lie above the disk sequence for $\mathrm{B}-\mathrm{V}>1.65$.

Optical and near-infrared color-color diagrams for M-dwarfs have recently been extensively discussed by Leggett (1992). In Figure 9 we show the V-I vs. B-V diagram for the objects we have classified. The well-known tendency for metal-poor M-subdwarfs to have redder B-V at a given V-I (e.g., Mould and McElroy 1978; Dahn et al. 1995) is clearly evident, for $V-I>1.7$. The

offset appears to be strongly related to metallicity, as the esdM are approximately 0.3 magnitudes redder than the disk stars in B-V with our sdM lying in between the two sequences. A V-I vs. $\mathrm{B}-\mathrm{V}$ diagram is therefore useful in identifying red subdwarfs and extreme subdwarfs. However, giants also lie redwards in B-V at a given V-I (Weistrop 1977). Indeed, Reid (1982) suggested on the basis of optical (BVRI) colors that Sm 183 might be a subdwarf similar to Kapteyn's star, but also noted the possibility that it could be a giant. In our spectrum, the $\mathrm{CaH}$ indices are weak compared to $\mathrm{TiO}$, implying that it is a low surface gravity giant.

\subsection{Tests of the Spectroscopic Metallicity Scale}

Theoretical structure predictions and HST globular cluster observations provide checks on our spectroscopic metallicity scale. We compare with broadband colors and magnitudes for $[m / H]=0.0,-0.5$, and -1.5 computed with stellar interior models that use the Allard and Hauschildt model atmospheres as outer boundary conditions (Baraffe et al. 1995). The tracks are 
shown in Figure 10. Baraffe et al. (1996) did not compute $[m / H]=-1.0$ models, but they did note that for $[m / H]=-2.0$ the models are shifted blueward by $\sim 0.2$ magnitudes with respect to the -1.5 track. Also shown is an even more recent set of solar metallicity models computed with the latest Allard and Hauschildt NextGen models (Baraffe and Chabrier 1996). The results dramatically illustrate the importance of boundary conditions - at $0.3 M_{\odot}\left(M_{V}=11.31, \mathrm{~V}-\mathrm{I}=2.19\right.$, $\left.T_{\text {eff }}=3403\right)$, the newer model is $201 \mathrm{~K}$ hotter, 1.21 magnitudes brighter in $M_{V}$, and 0.63 magnitudes bluer in V-I. As a result, the newer models fail to match observations (suggesting that they would be a poor choice to use for the $M_{V}$-Mass relation). In our HR diagram, the earlier set of computations give better agreement with the Population I sequence, although none of them convincingly duplicate the structure seen. As it stands, these models support the identification of the esdM with $[m / H] \sim-1.5$ to -2.0 and the sdM as more metal poor than -0.5 . Given the change of the theoretical models in less than one year, effective temperatures, metallicities, and masses derived from evolutionary models are clearly very uncertain - but the use of Allard and Hauschildt's extended model grid leads to consistent results for both spectroscopic and HR diagram metallicities.

HST photometry of a stellar cluster yields both a luminosity function and a color-magnitude diagram. Santiago et al. (1996, hereafter SEG) provide piecewise linear fits to the HR diagram of the clusters M15 $([m / H]=-2.26), \omega$ Cen (-1.6), 47 Tuc (-0.6), NGC 2420 (-0.45), and NGC 2477 (0.0), which are shown in Figure 11 superimposed on our data (including the earlier subdwarfs). The globular cluster data reach only the brightest of the sdM and esdM. The offsets however appear to be consistent with the esdM having abundances between -1.6 and -2.26 and the sdM between -1.6 and -0.6, as deduced for our spectroscopic analysis. SEG note that the slopes of their CMD are steeper than the nearby field parallax subdwarfs and attribute this to either calibration errors in the HST photometry or real astrophysical differences. The considerable difficulties in transforming HST magnitudes to the Cousins system are discussed by Harris et al. (1991) and Holtzman et al. (1995). In particular, the HST F606W filter includes a substantial fraction of the $R_{C}$ passband. However, the differences in slope of the main sequence are less evident in Figure 11 than in the fits reported by SEG in the region of overlap. It appears likely that the confusion in the definition of subdwarfs led the the inclusion of progressively less metal-poor stars at redder colors in the Richer and Fahlman (1992) main sequence, in particular stars with V-I $\gtrsim 2.4$ which we argue below are Intermediate Population II $([m / H] \sim-0.6)$ and which, in any case, are clearly much fainter and redder than the stars used to define the globular cluster slopes in the HST study. The fit to the NGC 2477 lower main sequence appears to lie above the local stars - perhaps due to color terms but perhaps also because of the inclusion of binaries or non-cluster members in the fits.

Most of the cool extreme subdwarfs in this paper come from the M92 USNO CCD parallaxes. Our spectroscopic metallicity scale is in agreement with the M92 estimate that the mean $[\mathrm{m} / \mathrm{H}]$ of extreme subdwarfs is $\sim-1.7$. Some authors have disputed the argument that the extreme subdwarfs represent the 'typical' halo metallicity of $\sim-1.7$. Richer and Fahlman (1992) argue 
that the extremely hot kinematics (15 of the 17 M92 extreme subdwarfs have $v_{\text {tan }}>275 \mathrm{~km} \mathrm{~s}^{-1}$ and the 5 stars with known total space velocities have $\left\langle v_{t o t}\right\rangle=427 \mathrm{~km} \mathrm{~s}^{-1}$ ) may point to their being more metal poor than the typical Population II stars. However, since there is no metallicity-velocity relation within the higher mass stars of the Population II halo (Norris 1986) this argument seems unconvincing. Note however that the Richer and Fahlman color-magnitude relation matches our sdM at $\mathrm{V}-\mathrm{I} \sim 1.5$ but matches the disk sequence by $V-I \sim 3$, which may be appropriate for their sample since their "Population II" luminosity function is in fact dominated by the IPII at the faint end (Reid et al. 1996). Lacking a direct determination of metallicity, Eggen (1996) estimated values of -2.5 to -3.5 for the M92 extreme subdwarfs but considered these values "guesses only."

\subsection{Where are the cool sdM?}

M92 note that their HR diagram (their Figure 10) shows a remarkable gap between the extreme subdwarfs and the disk sequence. In their HR diagram, only one subdwarf appears between the two sequences (at about V-I of 2.2). In our sample, there is clearly a continuous distribution for stars with $\mathrm{V}-\mathrm{I}<2.2$, leaving the gap for $2.2<\mathrm{V}-\mathrm{I}<2.8$. There are three major solutions to the gap, each of which was addressed by M92. First, the gap may be an illusion caused by stellar astrophysics - a continuous distribution in metallicity may not necessarily correspond to a simple distribution in a given observational HR diagram. Second, the gap may be due to selection effects such that stars in the gap are not chosen for parallax programs. Finally, it may represent a real lack of stars with metallicities between the disk sequence and the extreme subdwarf sequence. We will reconsider each of these options. It is certainly true that bandpass effects and the properties of cool atmospheres are important in interpreting HR diagrams. In Figure 8, we have seen that the behavior of the B-V HR diagram is opposite to that of the V-I diagram - at the reddest colors, the subdwarfs lie above the disk sequence, and the esdM lie above the sdM. A second example of the importance of bandpass effects is in the V-I HR diagram, where it appears that for the very coolest disk stars (LHS 2924 and 2065) V-I turns around, and becomes bluer with decreasing temperature (Bessell 1991; M92). Nevertheless, there is no evidence at present that the gap is caused by bandpass effects because M92 report the Allard model atmospheres do not produce such an effect. M92 consider that it is "very unlikely" that the gap is due to the USNO selection criteria, and therefore offer the preliminary interpretation that stars with intermediate metallicities are rare in the solar neighborhood. Since this result is quite surprising, we will next reconsider the selection effects that may entered this sample.

There necessarily were important selection effects in the catalog, since as discussed in Section 2, even in a proper motion survey the majority of stars will not be extremely metal poor or halo stars. It is difficult to know exactly what the selection effects entering the catalog were, but early studies that emphasized the targets surely were influential. Some of the M92 stars were never studied before (according to SIMBAD), but others had already been noted as interesting. 
Two of their stars (LHS 3382 and LHS 489) were pointed out by Ake and Greenstein (1980) as being spectroscopically much more metal poor than other known subdwarfs. Liebert et al. (1979) reported "sdM" spectra for five others (LHS 192, 197, 205a, 207, and 453) from a search for nearby degenerate stars - their Figure 2 presents a noisy spectrum of LHS 205a with strong hydride bands and a lack of TiO. Four others (as well as LHS 3382 again) were chosen by Hartwick et al. (1984) for having extremely large reduced proper motions, defined as

$$
H=M+5 \log v_{\text {tan }} / 4.74=m+5 \log \mu+5
$$

The lack of the sdM is obvious in their reduced proper motion diagram (their Figure 5). In contrast, there is no obvious gap in the reduced proper motion diagram (based on $\mathrm{B}$ and $\mathrm{R}$ magnitudes from the POSS plates) of the entire LHS catalog presented by Dawson (1986, his Figure 7). One explanation of the very large tangential velocities of the M92 sample $\left.\left(<v_{\text {tan }}\right\rangle=380 \mathrm{~km} \mathrm{~s}^{-1}\right)$ is that there was a implicit selection effect favoring large H. If so, a group of subdwarfs $\sim 1$ magnitude more luminous but the same reduced proper motions would have $\left.<v_{\text {tan }}\right\rangle=600 \mathrm{~km} \mathrm{~s}^{-1}$. Since this is greater than the escape velocity of the galaxy $\left(500 \mathrm{~km} \mathrm{~s}^{-1}\right.$, Carney et al. 1988)), these subdwarfs could not enter the sample. Similarly, the (unexplained) lower limit of $v_{t a n}=275 \mathrm{~km} \mathrm{~s}^{-1}$ would correspond to a lower limit of $v_{t a n}=435 \mathrm{~km} \mathrm{~s}^{-1}$. We therefore suggest that such a selection effect (as well as spectral selection of very weak TiO lines) would explain the lack of intermediate metallicity stars. As noted above, these stars are too faint to have been included in the more completely studied Giclas catalog which includes many bright early sdM. In any case, there are some likely LHS Catalog red sdM in the study of Hartwick et al. (1984) which do not yet have parallaxes - they identified both Class H stars (e.g., LHS 192, 1970, 3259, 3382, and 3548), which we call esdM, and Class I stars (e.g., LHS 29 and 64) which we can identify as sdM stars. They found three faint class I stars: LHS 2533, 2630, and 3189. We confirm the latter as an sdM5.0 star. Further observations of these three stars would greatly increase the information available on cool sdM stars.

\subsection{Kinematics}

Using the trigonometric parallaxes in Table \&, the radial velocities in Table 2, and the LHS catalog proper motion values, we can compute the U,V,W components of galactic velocity (Table 5). The errors in radial velocity, $\pm \sim 20 \mathrm{~km} \mathrm{~s}^{-1}$, are comparable to the uncertainties due to the trigonometric parallax errors. The kinematics of the sample are not representative of the Population II due to the uncertain selection criteria, but they can provide some information on the selection criteria used. Mean values and standard deviations (velocity dispersions) for the spectroscopic classes are listed in Table 6. The sdM, with the mean velocity component $\langle V\rangle=-202 \mathrm{~km} \mathrm{~s}^{-1}$, and the esdM, with a mean galactic velocity component $<V>=-287 \mathrm{~km} \mathrm{~s}^{-1}$, have clear Population II kinematics (the mean $V$ velocity for the halo is disputed but lies between -270 and $-180 \mathrm{~km} \mathrm{~s}^{-1}$ according to Majewski 1993). Our candidate halo stars that were classified as M V have both smaller $\langle V\rangle=-134 \mathrm{~km} \mathrm{~s}^{-1}$ and smaller $\mathrm{U}$ 
and $\mathrm{V}$ velocity dispersions - more typical of the IPII and certainly much greater than the disk $\left(<V>=-22 \mathrm{~km} \mathrm{~s}^{-1}\right)$. These non-subdwarfs in fact have bandstrength indices similar to the majority of RHG nearby stars with $V_{\text {tan }}>100 \mathrm{~km} \mathrm{~s}^{-1}$, which should be an IPII dominated sample. For both sdM and esdM, $\sigma_{U}$ and $\sigma_{W}$ are somewhat greater and $\sigma_{V}$ is somewhat less than the halo values of Norris (1986) as expected for a sample selected by large velocity but excluding near solar $\mathrm{V}$ values. Also given in Table 6 are the velocity dispersions for late M-dwarf RHG stars with $\mathrm{TiO} 5<0.4$ and either weak $\mathrm{CaH} 1$ (CaH1 $\geq 0.74$ ) or strong $\mathrm{CaH} 1$ (CaH1 < 0.74), as well as the RHG values for nearby disk M-dwarfs. The strong CaH1 stars have larger velocity dispersions, suggesting that they are older and supporting the idea they are more metal poor but their velocities are much less than a Population II group. Thus although CaH1 is useless as a temperature indicator for stars cooler than M3, it may prove to be useful metallicity indicator for late-type (M4 to M6.5) disk stars, particularly if $\mathrm{CaH}$ features can be reproduced by future model atmospheres.

We have also computed total galactocentric velocity for the stars after correcting for the Sun's motion with respect to the Local Standard of Rest (Mihalas and Binney 1981) and assuming a

LSR circular velocity of $220 \mathrm{~km} \mathrm{~s}^{-1}$. All of the stars are easily bound to Galaxy - the fastest sdM (LHS 467, $v_{\text {Gal }}=342 \mathrm{~km} \mathrm{~s}^{-1}$ ) and the fastest esdM (LHS 3548, $v_{\text {Gal }}=364 \mathrm{~km} \mathrm{~s}^{-1}$; LHS 453,

$v_{\text {Gal }}=451 \mathrm{~km} \mathrm{~s}^{-1}$ ) are comfortably less than the galactic escape speed of $\sim 500 \mathrm{~km} \mathrm{~s}^{-1}$ (Carney et al. 1988).

\section{Notes on Individual Stars}

\subsection{Dra (LHS 421)}

CM Dra (Gl 630.1) is a short period eclipsing binary with component masses $M_{A}=0.2307 \pm 0.0010$ and $M_{B}=0.2136 \pm 0.0010 M_{\odot}$ and helium abundance $Y_{A}=0.32$ and $Y_{B}=0.31 \pm 0.04$ ( Metcalfe et al. 1996). Based on the large W velocity and the observed low flaring rate and lacking a spectroscopic metallicity, Metcalfe et al. 1996 interpret the system as Population II and therefore interpret the high helium abundance as possible support for a higher primordial helium abundance. Using the observed spectrum (taken from RHG), we find that the system not very metal poor, with a best fit model of $[m / H]=0.0$, and does not show any CaH excess compared to Population I stars; however, infrared photometry (Leggett 1992) suggests it is more metal-poor than most disk stars. We therefore conclude CM Dra formed from significantly enriched material. The CNS3 W velocity of -34 ( -27 when corrected for the solar motion given in Mihalas and Binney 1981) implies that its maximum height above the plane is only $\sim 350$ pc (Kuijken and Gilmore 1989), whereas starcounts show the true Population II does not become dominant until beyond 5 kpc (Majewski 1993), so CM Dra can probably be considered an Intermediate Population II, or even Population I, star. 


\subsection{Barnard's Star (LHS 57) and Gl 299 (LHS 35)}

M92 have discussed the well known Barnard's Star (Gl 699) extensively. It lies perhaps 1.6 magnitudes below their mean disk main sequence, yet it is well known that spectroscopically it is an M-dwarf, a result that this study confirms. We believe that the distance below the main sequence is "artificially" enhanced by the presence of the kink in the main sequence (Gizis and Reid 1996 Figure 5) which is not fit well by a line. At spectral type M4.5 the disk sequence, when restricted to only single stars with high quality parallaxes and photometry, shows a sharp 1 magnitude drop. Hence Barnard's Star is only about 0.6 magnitudes below the disk sequence. It seems likely that Barnard's Star has a metallicity between -0.5 and -1.0 . It is a weak outlier in $\mathrm{TiO}$ and $\mathrm{CaH}$. The well known star Gl 299 (LHS 35) is quite similar in both its indices and absolute magnitude. We identify both as Intermediate Population II stars and as cool analogues to the spectroscopically unremarkable stars (e.g., LHS 301 and 376). The M4.5 V star LHS 3684 which has an M92 parallax is an only slightly cooler counterpart of Barnard's Star in both the spectroscopic indices and the HR diagram.

\subsection{The sdMe (LHS 482 and 2497) and Unresolved Binaries}

Two subdwarfs in this study show noticeable $H \alpha$ emission. Gl 781 (LHS 482) shows large radial velocity variations typical of a short period binary (Joy 1947). We have obtained echelle

spectra that confirm the radial velocity variations but do not show lines from the secondary Gizis et al.1996). We therefore have not adjusted the absolute magnitude in Table@ for the secondary component. Gl 455 (LHS 2497) shows weak H alpha emission (Hawley et al. 1996) and our recent echelle spectra have shown the system to be a short period double-lined spectroscopic binary (Gizis et al.1996). Since the system is near-equal luminosity we have not adjusted the color but have subtracted 0.75 from the absolute magnitude in Table 1 . The system is a strong outlier in Figure 1, yet even with the adjustment it has an absolute magnitude and color similar to the subluminous IPII dM stars. The YPC parallax is based upon two determinations with good agreement and has an uncertainty of less than $10 \%$. If it is not in error, perhaps the chromospheric activity affects the spectrum or magnitude of this interesting system. Unfortunately we have no other examples of late sdM that is comparable to this system. Young et al. (1987) argue that M-dwarf binaries with short periods ( $P \lesssim 5$ days) must show emission. The observed radial velocity variations are consistent with this limit. Mass determinations would provide a important constraint on theories of metal poor low-mass stars, but the short periods imply small separations ( $a \lesssim 4$ milliarcseconds) for these systems. No other sdM or esdM show $H \alpha$ emission in this study, and indeed no extreme M-subdwarfs with emission are yet known.

The sdMe discussed above are the only stars in the sample known to have unresolved companions. LHS 169, also known as Gl 129 but no longer within the nearby star catalog (CNS3) due to an improved parallax, has been reported to be spectroscopic binary. Three measurements 
of the radial velocity (Joy 1947) found radial velocities of $-139.6,-91.0$ and $-75.9 \mathrm{~km} \mathrm{~s}^{-1}$ with probable error $2.1 \mathrm{~km} \mathrm{~s}^{-1}$. The radial velocity variations have evidently never been confirmed or refuted since then, and Dawson and De Robertis (1988) suggest that the Joy (1947) velocities may be incorrect for a number of subdwarfs. LHS 64 has been reported to have velocity varying between -292 and -242 by Joy (1947) but we have obtained 3 echelle spectra which are consistent with no variations at all. Lacking any further data, we do not apply any correction to the absolute magnitude of either star for any companion. Presumably at least a few of the stars in our sample have unresolved companions that are still unknown, which could affect the magnitudes or radial velocities in a few cases.

\subsection{The most extreme subdwarf: LHS 453}

One important use of a classification system is identifying unusual stars. Our indices have allowed us to identify LHS 453 as even more metal poor than the other extreme subdwarfs. We would therefore expect this star to be below the other stars in the HR diagram, but the M92 observations $\left(\mathrm{V}-\mathrm{I}=2.22, M_{V}=13.08\right)$ place the star in the midst of the extreme subdwarf sequence. However, the reported mean error in $M_{V}$ is still fairly large at \pm 0.20 magnitudes. Perhaps coincidentally, this most metal poor star also has the largest galactocentric velocity in the sample, though Dawson and De Robertis (1988) showed that despite the large tangential velocity (M92 give $466.9 \pm 42.8$ ) the star is bound to the galaxy. If the parallax is indeed reduced, the tangential velocity would also be decreased. Alternatively, if LHS 453 is a binary, its $M_{V}$ would be fainter by up to 0.75 magnitudes. The best fit synthetic spectrum has $[m / H]=-2.5$, but we note that the synthetic $[m / H]=-3.0$ spectrum is almost identical. Although TiO is absent, LHS 453 is not likely to be more metal poor than this because the observed atomic lines are prominent and similar in strength to those of LHS 205a (which is only slightly later type in V-I color and $\mathrm{CaH}$ indices). According to the models, the atomic lines should be much weaker or absent for $[m / H]=-3.5$ or -4 at all temperatures.

\section{Conclusions}

This paper presents spectroscopy of 50 late-type stars that are significantly more metal-poor

than the Population I. Bandstrength indices are used to measure $\mathrm{TiO}$ and $\mathrm{CaH}$ features and show that many different metallicities can be distinguished in the Population II M stars, as expected from the metallicity spread seen in G-subdwarfs. The TiO feature at $\sim 7100 \AA$ is shown to put all the extreme subdwarfs into only a few spectral subclasses (K7 and M0). Instead, a $\mathrm{CaH}$ index which reproduces the Population I spectral classification system of Kirkpatrick, Henry, and McCarthy (1991) is used to classify stars. Based on the differences between the (weak) TiO and the $\mathrm{CaH}$ the metal poor $\mathrm{M}$ stars are divided into the simple categories of M-subdwarfs (sdM) and extreme M-subdwarfs (esdM) and spectral standards are presented (Figures 0 and 3). 
Synthetic spectra computed by Allard and Hauschildt (1995) are fit to the observed spectra. Metallicities of $[m / H] \sim-2$ are derived for the esdM and $\sim-1.2$ for the sdM. These estimates are in agreement with the estimates of G89 and M92 but not those of Richer and Fahlman (1992) or Eggen (1996). HR diagrams based on literature observations are presented for dM, sdM and esdM. They are at least qualitatively in agreement with theoretical computations and HST observations of globular clusters. However, the former are sensitive to the boundary conditions used and the latter are likely to suffer from large color terms. The agreement of all three methods seems to indicate the metallicity scale is accurate to $\sim 0.5$ dex.

Given this metallicity scale, we discuss a number of interesting subdwarfs and stars that have been called subdwarfs. The eclipsing binary system CM Dra is shown to be significantly enriched such that its composition is not likely to reflect the primordial helium abundance. The "old disk subdwarfs" such as Gl 299 and Gl 699 are probably not as subluminous as is usually thought taking into account the detailed structure of the HR diagram, rather than simply fitting a straight line, they are only $\sim 0.6$ magnitudes subluminous rather than the usually cited $\sim 1.2$ magnitude. This naturally explains the only slight spectral differences, which are shown to be similar to differences of hotter M-dwarfs that are also $\sim 0.6$ magnitudes subluminous. Finally, we show that LHS 453 is more metal poor than the other extreme subdwarfs with $[\mathrm{m} / H] \sim-2.5$ to -3 .

I would like to thank Neill Reid for helpful comments and help with the observations. The referee, Todd Henry, provided comments which substantially improved this paper. I am indebted to France Allard and Peter Hauschildt for making their synthetic spectra available. I am grateful for Greenstein and Kinsley Fellowships and partial support through NASA grant GO-5353.0-93A. This research has made use of the Simbad database, operated at CDS, Strasbourg, France.

Table 1. Spectroscopic Indices

\begin{tabular}{cccc}
\hline \hline Band & S1 & W & S2 \\
\hline TiO 5 & $7042-7046$ & $7126-7135$ & \\
CaH 1 & $6345-6355$ & $6380-6390$ & $6410-6420$ \\
CaH 2 & $7042-7046$ & $6814-6846$ & \\
CaH 3 & $7042-7046$ & $6960-6990$ & \\
\hline
\end{tabular}


Table 2. Spectroscopic Observations

\begin{tabular}{|c|c|c|c|c|c|c|c|c|}
\hline LHS & Name & TiO5 & $\mathrm{CaH} 1$ & $\mathrm{CaH} 2$ & $\mathrm{CaH} 3$ & $v_{\text {rad }}$ & class & Source \\
\hline 12 & G 003-036 & 0.894 & 0.852 & 0.746 & 0.878 & 25.1 & sdM0.0 & 60 \\
\hline 20 & GJ 1062 & 0.677 & 0.708 & 0.499 & 0.710 & -104.3 & sdM2.5 & RHG \\
\hline 29 & Kapteyn's & 0.810 & 0.778 & 0.594 & 0.792 & 242.8 & sdM1.0 & 100 \\
\hline 42 & Ross 451 & 0.925 & 0.871 & 0.757 & 0.885 & -148.0 & sdM0.0 & 60 \\
\hline 55 & GJ 1200 & 0.439 & 0.816 & 0.420 & 0.705 & -21.2 & M3.5 V & 60 \\
\hline 57 & Barnard's & 0.394 & 0.752 & 0.394 & 0.652 & -120.2 & $\mathrm{M} 4.0 \mathrm{~V}$ & 60 \\
\hline 61 & Gl 817 & 0.645 & 0.823 & 0.582 & 0.799 & 25.9 & M1.5 V & 60 \\
\hline 64 & Wo 9722 & 0.773 & 0.731 & 0.572 & 0.754 & -235.5 & sdM1.5 & RHG \\
\hline 104 & G $030-048$ & 1.009 & 0.911 & 0.854 & 0.938 & -173.8 & esdK7 & 60 \\
\hline 156 & G 004-029 & 0.655 & 0.648 & 0.445 & 0.659 & -42.5 & sdM3.0 & 100 \\
\hline 161 & G $075-047$ & 0.964 & 0.739 & 0.515 & 0.751 & -35.1 & esdM2.0 & 100 \\
\hline 169 & G $005-022$ & 1.008 & 0.879 & 0.862 & 0.951 & -119.2 & esdK7 & 60 \\
\hline 170 & G 078-026 & 1.007 & 0.986 & 0.931 & 0.961 & -183.2 & sdK & 60 \\
\hline 173 & G 038-001 & 0.961 & 0.942 & 0.868 & 0.938 & -159.2 & $\operatorname{sdK} 7$ & 60 \\
\hline 174 & G $037-040$ & 0.877 & 0.817 & 0.686 & 0.833 & -225.2 & sdM0.5 & 60 \\
\hline 178 & G 079-059 & 0.674 & 0.766 & 0.551 & 0.760 & -59.7 & sdM1.5 & 60 \\
\hline 182 & G 095-059 & 0.975 & 0.842 & 0.735 & 0.843 & -238.1 & esdM0.0 & 60 \\
\hline 185 & G 007-017 & 0.957 & 0.737 & 0.659 & 0.795 & 37.7 & esdM0.5 & 60 \\
\hline 192 & LP 302-31 & 0.946 & 0.710 & 0.603 & 0.768 & 79.9 & esdM1.0 & 200 \\
\hline $205 a$ & LP $417-44$ & 0.889 & 0.512 & 0.347 & 0.513 & -76.4 & esdM4.5 & 200 \\
\hline 211 & G 099-033 & 0.796 & 0.797 & 0.703 & 0.837 & -129.2 & sdM0.0 & 100 \\
\hline 216 & G $105-023$ & 0.725 & 0.743 & 0.496 & 0.732 & 392.5 & sdM2.0 & 60 \\
\hline 218 & G 103-046 & 0.500 & 0.759 & 0.413 & 0.686 & -20.4 & M3.5 V & 100 \\
\hline 236 & G 251-044 & 0.980 & 0.944 & 0.879 & 0.931 & 66.8 & sdK7 & 60 \\
\hline 254 & LP $666-11$ & 0.178 & 0.678 & 0.230 & 0.517 & 20.2 & M6.5 V & 100 \\
\hline 272 & LP $788-27$ & 0.678 & 0.676 & 0.441 & 0.669 & 264.4 & sdM3.0 & 100 \\
\hline 276 & G 117-061 & 0.686 & 0.888 & 0.623 & 0.828 & 46.9 & M1.0 V & 60 \\
\hline 301 & GJ 1146 & 0.481 & 0.773 & 0.431 & 0.683 & 94.4 & M3.5 V & 60 \\
\hline 307 & G $176-040$ & 0.910 & 0.763 & 0.671 & 0.837 & -64.8 & sdM0.5 & 60 \\
\hline 320 & G 011-035 & 0.620 & 0.756 & 0.512 & 0.751 & 81.1 & sdM2.0 & 60 \\
\hline 343 & G 061-021 & 1.001 & 0.953 & 0.901 & 0.937 & 165.5 & sdK & 60 \\
\hline 364 & G $165-047$ & 0.979 & 0.699 & 0.604 & 0.740 & 12.8 & esdM1.5 & 60 \\
\hline 375 & LP 857-48 & 0.829 & 0.499 & 0.372 & 0.547 & 181.2 & esdM4.0 & 60 \\
\hline 376 & G $135-067$ & 0.448 & 0.795 & 0.423 & 0.682 & -19.6 & M3.5 V & 60 \\
\hline 377 & LP 440-52 & 0.232 & 0.567 & 0.205 & 0.396 & 179.7 & sdM7.0 & 200 \\
\hline 407 & LP $803-27$ & 0.601 & 0.584 & 0.351 & 0.528 & -173.1 & sdM5.0 & 60 \\
\hline
\end{tabular}


Table 2-Continued

\begin{tabular}{|c|c|c|c|c|c|c|c|c|}
\hline LHS & Name & $\mathrm{TiO} 5$ & $\mathrm{CaH} 1$ & $\mathrm{CaH} 2$ & $\mathrm{CaH} 3$ & $v_{\text {rad }}$ & class & Source \\
\hline 410 & G 016-018 & .724 & 0.822 & 0.612 & 0.814 & 10.5 & M1.0 V & 60 \\
\hline 418 & G $138-025$ & 0.941 & 0.908 & 0.814 & .910 & -14.4 & $\mathrm{~K} 7 \mathrm{~V}$ & 60 \\
\hline 425 & G 138-059 & 0.646 & 0.762 & 0.503 & 0.738 & -49.3 & sdM2.0 & 60 \\
\hline 453 & LP 139-14 & 1.070 & 0.514 & 0.407 & 0.590 & 32.1 & esdM3.5 & 200 \\
\hline 460 & GJ 1225 & 0.337 & 0.736 & 0.321 & 0.603 & -53.2 & M5.0 V & 60 \\
\hline 467 & G 021-023 & 0.966 & 0.898 & 0.786 & 0.897 & 183.6 & sdK7 & 60 \\
\hline 479 & G 142-052 & 0.792 & 0.768 & 0.610 & 0.801 & -75.4 & & 60 \\
\hline 482 & Gl 781 & 0.774 & 0.718 & 0.558 & 0.746 & -107.6 & sdM1.5 & RHG \\
\hline 489 & LP515-3 & 0.998 & 0.853 & 0.766 & 0.889 & -95.3 & esdM0.0 & 60 \\
\hline 491 & G 210-019 & 0.843 & 0.708 & 0.582 & 0.759 & -142.9 & $\mathrm{sdN}$ & 60 \\
\hline 522 & G 018-051 & 1.024 & 0.926 & & 0.929 & -159.8 & & 60 \\
\hline 536 & G 12 & & & & & -57.8 & & 60 \\
\hline 537 & G $028-043$ & 1.002 & 1.010 & 0.978 & 0.982 & -113.0 & K5 V & 60 \\
\hline 1088 & G 217-055 & 0.454 & 0.781 & 0.427 & 0.740 & 17.5 & M3.5 V & 60 \\
\hline 1164 & L $220-27$ & 0.654 & 0.868 & 0.988 & 0.955 & -22.5 & K5 V & 100 \\
\hline 1174 & LP 406-47 & 0.924 & 0 . & 0 & 59 & -111.7 & esdM3.0 & $200^{\mathrm{a}}$ \\
\hline 1481 & LP 711-32 & 0.554 & 0.708 & 0.443 & 0.681 & 94.5 & sdM3.0 & RHG \\
\hline $1742 \mathrm{a}$ & LP $417-42$ & 0.689 & 0.428 & 0.284 & 0.429 & 218.1 & esdM5.5 & 200 \\
\hline 1970 & LP 484-6 & 0.878 & 0.5 & 0.475 & 0.657 & 9.8 & & 200 \\
\hline 2110 & LP $787-4$ & 0.362 & 0.747 & 0.366 & 0.653 & 37.8 & $\mathrm{M} 4.0 \mathrm{~V}$ & 100 \\
\hline 2497 & Gl 455 & 0.542 & 0.656 & 0.400 & 0.627 & 36.2 & sdM3.5 & RHG \\
\hline 2715 & Gl 506.1 & 0.994 & 1.019 & 0.971 & 0.975 & 36.0 & $\operatorname{sdK}$ & 60 \\
\hline 2852 & LP 856-36 & & & & & 13.3 & & RHG \\
\hline 3061 & LP 502-32 & 0.737 & 0.497 & 0.292 & 0.497 & 65.4 & esdM5.0 & 200 \\
\hline 3073 & G 137-008 & 0.989 & 0.918 & 0.869 & 0.922 & -254.5 & sdK7 & 60 \\
\hline 3084 & G 015-026 & 0.840 & 0.878 & 0.724 & 0.857 & -43.8 & $\operatorname{sdK}$ & 60 \\
\hline 3189 & LP $225-22$ & 0.341 & 0.637 & 0.305 & 0.579 & -106.2 & sdM5.0 & 200 \\
\hline 3192 & Gl 871 & 0.454 & 0.768 & 0.433 & 0.706 & 0.6 & & 60 \\
\hline 3193 & G 169-007 & 0.688 & 0.878 & 0.635 & 0.829 & 20.1 & M1.0 V & 60 \\
\hline 3259 & LP 686-36 & 0.995 & 0.811 & 0.657 & 0.803 & -217.0 & esdM0.5 & 200 \\
\hline 3382 & LP 24-219 & 0.937 & 0.635 & 0.485 & 0.667 & -138.9 & esdM2.5 & $200^{\mathrm{a}}$ \\
\hline 3409 & LP $141-1$ & 0.443 & 0.645 & 0.355 & 0.587 & -80.3 & sdM4.5 & RHG \\
\hline 3480 & LP 869-24 & 0.458 & 0.695 & 0.363 & 0.632 & 12.9 & sdM4.0 & 200 \\
\hline 3481 & LP 753-21 & 0.782 & 0.669 & 0.467 & 0.694 & 75.4 & sdM2.5 & 200 \\
\hline 3548 & LP 695-96 & 0.886 & 0.839 & 0.435 & 0.660 & 80.6 & esdM3.0 & $200^{\mathrm{a}}$ \\
\hline 3628 & LP 757-13 & 0.908 & 0.643 & 0.576 & 0.742 & 10.1 & esdM1.5 & 100 \\
\hline
\end{tabular}


Table 2-Continued

\begin{tabular}{clrrrrrrr}
\hline \hline LHS & \multicolumn{1}{c}{ Name } & TiO5 & CaH1 & CaH2 & CaH3 & $v_{\text {rad }}$ & class & Source \\
\hline 3684 & LP 518-12 & 0.377 & 0.733 & 0.344 & 0.637 & -75.3 & M4.5 V & 200 \\
3867 & G 067-030 & 1.024 & 0.997 & 0.962 & 0.982 & -108.8 & sdK & 60 \\
3957 & G 190-026 & 0.727 & 0.846 & 0.648 & 0.842 & -85.0 & M0.5 V & 60 \\
6304 & G 169-009 & 0.587 & 0.785 & 0.505 & 0.751 & -9.1 & M2.5 V & 60 \\
$\ldots$ & G 017-006 & 0.781 & 0.920 & 0.702 & 0.875 & 8.9 & M0.0 V & 60 \\
$\ldots$ & Sm 183 & 0.880 & 0.947 & 0.867 & 0.920 & -12.3 & gM & 100 \\
$\ldots$ & TVLM 832-42500 & 0.186 & 0.842 & 0.171 & 0.452 & 72.1 & $>$ M6.0 V & 200 \\
& & & & & & & & \\
\hline
\end{tabular}

a Observed with August 1995 setup

Table 3. Model Best Fits

\begin{tabular}{|c|c|c|c|c|c|c|c|c|c|c|c|}
\hline Star & Class & $T_{e f f}$ & {$[m / H]$} & Star & Class & $T_{e f f}$ & {$[m / H]$} & Star & Class & $T_{e f f}$ & {$[m / H]$} \\
\hline LHS 410 & M1.0 V & 3700 & -1.0 & LHS 307 & sdM0.5 & 3700 & -1.5 & LHS 3259 & esdM0.5 & 3700 & -2.0 \\
\hline LHS 301 & M3.5 V & 3700 & -0.5 & LHS 29 & sdM1.0 & 3700 & -1.5 & LHS 192 & esdM1.0 & 3600 & -2.5 \\
\hline LHS 218 & M3.5 V & 3600 & -1.0 & LHS 479 & sdM1.0 & 3700 & -1.0 & LHS 364 & esdM1.5 & 3600 & -2.5 \\
\hline LHS 3192 & M3.5 V & 3600 & -0.5 & LHS 178 & sdM1.5 & 3600 & -1.0 & LHS 3628 & esdM1.5 & 3600 & -1.5 \\
\hline LHS 376 & M3.5 V & 3600 & -0.5 & LHS 482 & sdM1.5 & 3600 & -1.0 & LHS 161 & esdM2.0 & 3600 & -2.0 \\
\hline LHS 6304 & $\mathrm{M} 2.5 \mathrm{~V}$ & 3600 & -1.0 & LHS 491 & sdM1.5 & 3600 & -1.5 & LHS 3382 & esdM2.5 & 3500 & -1.5 \\
\hline LHS 1088 & M3.5 V & 3500 & 0.0 & LHS 64 & sdM1.5 & 3600 & -1.0 & LHS 1970 & esdM2.5 & 3500 & -1.5 \\
\hline LHS 2110 & $\mathrm{M} 4.0 \mathrm{~V}$ & 3500 & 0.0 & LHS 320 & sdM2.0 & 3600 & -1.0 & LHS 1174 & esdM3.0 & 3500 & -1.5 \\
\hline LHS 55 & M3.5 V & 3500 & 0.0 & LHS 216 & sdM2.0 & 3600 & -1.0 & LHS 3548 & esdM3.0 & 3500 & -1.5 \\
\hline LHS 57 & $\mathrm{M} 4.0 \mathrm{~V}$ & 3500 & -0.5 & LHS 425 & sdM2.0 & 3600 & -1.0 & LHS 453 & esdM3.5 & 3400 & -2.5 \\
\hline LHS 3684 & $\mathrm{M} 4.5 \mathrm{~V}$ & 3500 & -0.5 & LHS 2852 & sdM2.0 & 3600 & -1.0 & LHS 375 & esdM4.0 & 3400 & -2.0 \\
\hline LHS 460 & M5.0 V & 3300 & 0.0 & LHS 20 & sdM2.5 & 3600 & -1.0 & LHS 205a & esdM4.5 & 3400 & -2.0 \\
\hline LHS 254 & M6.5 V & 3200 & 0.0 & LHS 3481 & sdM2.5 & 3600 & -1.5 & LHS 3061 & esdM5.0 & 3300 & -2.0 \\
\hline \multirow[t]{9}{*}{ TVLM 832-42500 } & $>\mathrm{M} 6.0 \mathrm{~V}$ & 3000 & 0.0 & LHS 272 & sdM3.0 & 3600 & -1.5 & LHS $1742 \mathrm{a}$ & esdM5.5 & 3300 & -2.0 \\
\hline & & & & LHS 156 & sdM3.0 & 3600 & -1.5 & & & & \\
\hline & & & & LHS 1481 & sdM3.0 & 3600 & -1.0 & & & & \\
\hline & & & & LHS 2497 & $\operatorname{sdM} 3.5$ & 3500 & -1.0 & & & & \\
\hline & & & & LHS 3480 & $\operatorname{sdM} 4.0$ & 3500 & -1.0 & & & & \\
\hline & & & & LHS 3409 & sdM4.5 & 3400 & -1.0 & & & & \\
\hline & & & & LHS 3189 & sdM5.0 & 3400 & -1.0 & & & & \\
\hline & & & & LHS 407 & sdM5.0 & 3400 & -1.5 & & & & \\
\hline & & & & LHS 377 & sdM7.0 & 3200 & -1.5 & & & & \\
\hline
\end{tabular}


Table 4. Photometry

\begin{tabular}{|c|c|c|c|c|c|c|c|c|c|c|}
\hline LHS & $\mathrm{V}$ & $\mathrm{B}-\mathrm{V}$ & $\mathrm{V}-\mathrm{R}$ & R-I & V-I & $\pi\left({ }^{\prime \prime}\right)$ & $\sigma_{\pi}\left({ }^{\prime \prime}\right)$ & $\Delta_{L K}^{\mathrm{a}}$ & $M_{V}$ & Source $^{b}$ \\
\hline 12 & 12.24 & 1.45 & 0.90 & 0.90 & 1.80 & 0.0359 & 0.0031 & -0.08 & 10.02 & $\mathrm{Y}, \mathrm{L}$ \\
\hline 20 & 13.01 & 1.68 & 1.03 & 1.17 & 2.20 & 0.0648 & 0.0025 & -0.01 & 12.07 & $\mathrm{Y}, \mathrm{L}$ \\
\hline 29 & 8.84 & 1.56 & 0.96 & 1.00 & 1.96 & 0.2583 & 0.0065 & -0.01 & 10.90 & $\mathrm{Y}, \mathrm{L}$ \\
\hline 35 & 12.83 & 1.72 & 1.25 & 1.67 & 2.92 & 0.1480 & 0.0022 & -0.00 & 13.68 & $\mathrm{Y}, \mathrm{L}$ \\
\hline 42 & 12.23 & 1.45 & 1.06 & 0.92 & 1.98 & 0.0327 & 0.0025 & -0.06 & 9.80 & Y,E79 \\
\hline 44 & 6.45 & 0.76 & $\ldots$ & $\ldots$ & $\ldots$ & 0.1127 & 0.0014 & -0.00 & 6.71 & $\mathrm{Y}, \mathrm{S}$ \\
\hline 52 & 9.43 & 0.84 & 0.52 & 0.49 & 1.01 & $\ldots$ & $\ldots$ & $\ldots$ & $\ldots$ & $\mathrm{R}$ \\
\hline 53 & 9.04 & 0.78 & 0.45 & 0.46 & 0.91 & $\ldots$ & $\ldots$ & $\ldots$ & $\ldots$ & $\mathrm{R}$ \\
\hline 55 & 12.90 & 1.54 & $\ldots$ & $\ldots$ & $\ldots$ & 0.0550 & 0.0029 & -0.03 & 11.60 & $\mathrm{HD}, \mathrm{S}$ \\
\hline 64 & 13.30 & 1.55 & $\ldots$ & $\ldots$ & $\ldots$ & 0.0419 & 0.0022 & -0.03 & 11.41 & $\mathrm{Y}, \mathrm{S}$ \\
\hline 103 & 14.16 & $\ldots$ & 1.15 & 1.47 & 2.62 & 0.0536 & 0.0021 & -0.02 & 12.81 & $\mathrm{Y}, \mathrm{WU}$ \\
\hline 104 & 13.78 & 1.34 & 0.81 & 0.91 & 1.72 & $\cdots$ & $\ldots$ & $\ldots$ & & E79 \\
\hline 137 & 13.36 & 1.60 & $\ldots$ & $\ldots$ & $\ldots$ & 0.0463 & 0.0027 & -0.03 & 11.69 & $\mathrm{Y}, \mathrm{S}$ \\
\hline 139 & 15.05 & 1.86 & 1.24 & 1.61 & 2.85 & 0.0485 & 0.0044 & -0.09 & 13.48 & Y,E79 \\
\hline 156 & 14.89 & 1.70 & 0.92 & 1.25 & 2.17 & 0.0277 & 0.0035 & -0.18 & 12.1 & Y,E79 \\
\hline 161 & 14.75 & 1.55 & 1.01 & 0.96 & 1.98 & 0.0260 & 0.0048 & -0.42 & 11.82 & Y,E79 \\
\hline 163 & 13.06 & 1.57 & 1.12 & 1.43 & 2.55 & 0.0496 & 0.0043 & -0.08 & 11.54 & $\mathrm{Y}, \mathrm{B}$ \\
\hline 169 & 14.13 & 1.45 & 0.91 & 0.76 & 1.72 & 0.0309 & 0.0023 & -0.06 & 11.58 & Y,L,E79 \\
\hline 170 & 10.69 & 1.22 & 0.80 & 0.70 & 1.50 & 0.0348 & 0.0025 & -0.05 & 8.40 & Y,E79 \\
\hline 173 & 11.12 & 1.31 & $\ldots$ & $\ldots$ & $\ldots$ & 0.0353 & 0.0031 & -0.08 & 8.86 & $\mathrm{Y}, \mathrm{S}$ \\
\hline 174 & 12.75 & 1.52 & 0.94 & 0.89 & 1.83 & 0.0204 & 0.0045 & -0.65 & 9.30 & Y,DF89 \\
\hline 175 & 9.91 & 0.65 & $\cdots$ & $\ldots$ & $\cdots$ & 0.0218 & 0.0022 & -0.11 & 6.6 & $\mathrm{Y}, \mathrm{R}$ \\
\hline 178 & 12.90 & 1.55 & 0.95 & 1.11 & 2.06 & 0.0451 & 0.0066 & -0.24 & 11.17 & Y,E79 \\
\hline 182 & 13.42 & 1.57 & $\ldots$ & $\ldots$ & $\ldots$ & 0.0234 & 0.0025 & -0.12 & 10.27 & $\mathrm{Y}, \mathrm{S}$ \\
\hline 185 & 15.30 & 1.79 & 0.98 & 0.85 & 1.83 & 0.0167 & 0.0046 & $\mathrm{~L}$ & 11.41 & Y,DF89 \\
\hline 192 & 17.33 & $\ldots$ & $\ldots$ & $\ldots$ & 1.98 & 0.0102 & 0.0008 & -0.06 & 12.37 & M92 \\
\hline $205 a$ & 18.93 & $\ldots$ & $\ldots$ & $\ldots$ & 2.35 & 0.0104 & 0.0013 & -0.17 & 14.02 & M92 \\
\hline 211 & 14.11 & 1.45 & 0.91 & 1.05 & 1.96 & 0.0188 & 0.0029 & -0.28 & 10.48 & $\mathrm{Y}, \mathrm{L}$ \\
\hline 216 & 14.66 & 1.62 & 0.99 & 1.09 & 2.08 & 0.0306 & 0.0030 & -0.10 & 12.09 & $\mathrm{Y}, \mathrm{L}$ \\
\hline 218 & 14.84 & 1.57 & 1.12 & 1.33 & 2.45 & 0.0296 & 0.0030 & -0.11 & 12.20 & Y,DF89 \\
\hline 232 & 13.72 & 1.15 & $\ldots$ & $\ldots$ & $\ldots$ & 0.0148 & 0.0025 & -0.34 & 9.57 & $\mathrm{Y}, \mathrm{S}$ \\
\hline 236 & 13.10 & 1.33 & $\ldots$ & $\ldots$ & $\ldots$ & 0.0187 & 0.0018 & -0.10 & 9.46 & $\mathrm{Y}, \mathrm{S}$ \\
\hline 241 & 8.32 & 0.62 & $\ldots$ & $\cdots$ & $\cdots$ & $\ldots$ & $\cdots$ & $\cdots$ & $\cdots$ & $\mathrm{S}$ \\
\hline 254 & 17.41 & 1.75 & 1.90 & 2.07 & 3.97 & $\ldots$ & $\ldots$ & $\ldots$ & $\ldots$ & $\mathrm{L}$ \\
\hline 272 & 13.46 & $\cdots$ & 1.34 & 1.36 & 2.70 & $\cdots$ & $\ldots$ & $\cdots$ & $\ldots$ & DF89 \\
\hline 276 & 11.86 & 1.43 & 0.93 & 1.04 & 1.97 & 0.0347 & 0.0025 & -0.05 & 9.56 & $\mathrm{Y}, \mathrm{B}$ \\
\hline
\end{tabular}


Table 4-Continued

\begin{tabular}{|c|c|c|c|c|c|c|c|c|c|c|}
\hline LHS & V & $\mathrm{B}-\mathrm{V}$ & V-R & R-I & V-I & $\pi\left({ }^{\prime \prime}\right)$ & $\sigma_{\pi}\left(^{\prime \prime}\right)$ & $\Delta_{L K}^{\mathrm{a}}$ & $M_{V}$ & Source $^{b}$ \\
\hline 301 & 13.57 & 1.57 & 1.08 & 1.39 & 2.47 & 0.0540 & 0.0036 & -0.04 & 12.23 & Y,W88,E79 \\
\hline 307 & 15.22 & 1.56 & $\cdots$ & $\ldots$ & 1.92 & 0.0183 & 0.0027 & -0.25 & 11.53 & $\mathrm{Y}, \mathrm{L}^{*}$ \\
\hline 320 & 14.00 & 1.54 & 0.88 & 1.26 & 2.14 & 0.0260 & 0.0034 & -0.19 & 11.07 & $\mathrm{Y}, \mathrm{E} 79$ \\
\hline 343 & 13.87 & 1.30 & 0.87 & 0.69 & 1.56 & 0.0167 & 0.0031 & -0.43 & 9.98 & $\mathrm{HD}, \mathrm{L}$ \\
\hline 364 & 14.61 & 1.71 & 1.03 & 0.92 & 1.95 & 0.0374 & 0.0037 & -0.10 & 12.47 & Y,DF92 \\
\hline 375 & 15.68 & 1.87 & 1.08 & 1.12 & 2.20 & 0.0395 & 0.0010 & -0.01 & 13.66 & $\mathrm{RA}$ \\
\hline 376 & 15.00 & 1.64 & 1.08 & 1.33 & 2.41 & 0.0207 & 0.0048 & -0.74 & 11.58 & $\mathrm{Y}, \mathrm{E} 79$ \\
\hline 377 & 18.39 & $\cdots$ & $\ldots$ & $\cdots$ & 3.48 & 0.0284 & 0.0008 & -0.01 & 15.66 & M92 \\
\hline 407 & 16.57 & 1.93 & 1.06 & 1.33 & 2.39 & 0.0315 & 0.0020 & -0.04 & 14.06 & $\mathrm{RA}$ \\
\hline 410 & 13.36 & $\ldots$ & 0.94 & 1.06 & 2.00 & 0.0240 & 0.0034 & -0.23 & 10.26 & Y,HD,W91 \\
\hline 418 & 13.51 & 1.42 & 0.88 & 0.80 & 1.68 & 0.0141 & 0.0033 & -0.76 & 9.26 & HD,La \\
\hline 420 & 7.30 & 0.54 & $\ldots$ & $\cdots$ & $\cdots$ & 0.0324 & 0.0057 & -0.37 & 4.85 & $\mathrm{Y}, \mathrm{S}$ \\
\hline 425 & 15.00 & 1.60 & $\ldots$ & $\ldots$ & $\ldots$ & 0.0261 & 0.0047 & -0.40 & 12.08 & $\mathrm{Y}, \mathrm{S}$ \\
\hline 453 & 18.02 & $\ldots$ & $\ldots$ & $\ldots$ & 2.22 & 0.0103 & 0.0009 & -0.08 & 13.08 & M92 \\
\hline 460 & 15.40 & 1.77 & 1.34 & 1.70 & 3.04 & 0.0544 & 0.0028 & -0.03 & 14.08 & Y,DF92 \\
\hline 467 & 12.21 & 1.43 & $\ldots$ & $\ldots$ & 1.70 & 0.0362 & 0.0020 & -0.03 & 10.00 & $\mathrm{Y}, \mathrm{L}$ \\
\hline 479 & 14.31 & 1.51 & $\ldots$ & $\ldots$ & $\ldots$ & 0.0224 & 0.0023 & -0.11 & 11.06 & $\mathrm{Y}, \mathrm{S}$ \\
\hline 482 & 11.98 & 1.56 & 0.95 & 1.04 & 1.99 & 0.0603 & 0.0017 & -0.01 & 10.88 & $\mathrm{Y}, \mathrm{L}$ \\
\hline 489 & 15.48 & 1.69 & 0.91 & 0.86 & 1.77 & 0.0189 & 0.0036 & -0.45 & 11.86 & Y,DF89 \\
\hline 491 & 14.70 & 1.68 & 0.98 & 0.98 & 1.96 & 0.0211 & 0.0036 & -0.35 & 11.32 & Y,DF92 \\
\hline 522 & 14.15 & 1.41 & 0.84 & 0.78 & 1.62 & 0.0268 & 0.0021 & -0.06 & 11.29 & $\mathrm{Y}, \mathrm{B}$ \\
\hline 536 & 14.65 & 1.55 & $\ldots$ & $\ldots$ & $\ldots$ & 0.0227 & 0.0025 & -0.13 & 11.43 & $\mathrm{Y}, \mathrm{S}$ \\
\hline 537 & 9.96 & 0.70 & $\ldots$ & $\ldots$ & $\ldots$ & $\ldots$ & $\ldots$ & $\ldots$ & $\ldots$ & $\mathrm{S}$ \\
\hline 1174 & 16.99 & $\ldots$ & $\ldots$ & $\ldots$ & 2.09 & 0.0157 & 0.0012 & -0.06 & 12.97 & M92 \\
\hline 1319 & 14.82 & 1.45 & $\ldots$ & $\ldots$ & $\ldots$ & $\ldots$ & $\ldots$ & $\ldots$ & $\ldots$ & $\mathrm{S}$ \\
\hline 1481 & 12.67 & 1.73 & 1.08 & 1.32 & 2.40 & $\ldots$ & $\ldots$ & $\ldots$ & $\ldots$ & CNS3,E87 \\
\hline 1555 & 13.90 & 1.70 & $\cdots$ & $\cdots$ & $\cdots$ & 0.0168 & 0.0019 & -0.14 & 10.03 & $\mathrm{Y}, \mathrm{S}$ \\
\hline $1742 \mathrm{a}$ & 18.80 & $\ldots$ & $\ldots$ & $\ldots$ & 2.74 & 0.0134 & 0.0012 & -0.08 & 14.44 & M92 \\
\hline 1970 & 17.76 & 1.68 & $\cdots$ & $\cdots$ & 2.09 & 0.0129 & 0.0008 & -0.04 & 13.31 & $\mathrm{M}, \mathrm{L}$ \\
\hline 2045 & 18.49 & $\ldots$ & $\ldots$ & $\ldots$ & 2.46 & 0.0111 & 0.0009 & -0.07 & 13.72 & M92 \\
\hline 2110 & 16.97 & $\ldots$ & $\cdots$ & $\ldots$ & 2.84 & 0.0151 & 0.0010 & -0.04 & 12.86 & M92 \\
\hline 2204 & 16.80 & $\ldots$ & $\ldots$ & $\ldots$ & 3.23 & 0.0288 & 0.0009 & -0.01 & 14.10 & M92 \\
\hline 2497 & 12.85 & 1.74 & 1.08 & 1.22 & 2.47 & 0.0493 & 0.0031 & -0.04 & $12.06^{\mathrm{c}}$ & $\mathrm{Y}, \mathrm{S}$ \\
\hline 2715 & 10.83 & 1.03 & 0.62 & 0.55 & 1.17 & 0.0286 & 0.0033 & -0.14 & 8.11 & $\mathrm{Y}, \mathrm{A}, \mathrm{AM}, \mathrm{B}$ \\
\hline 2852 & 12.15 & 1.70 & 1.06 & 1.22 & 2.18 & 0.0395 & 0.0174 & $\mathrm{~L}$ & 10.13 & Y,CNS3,E87 \\
\hline 3061 & 19.50 & $\cdots$ & $\cdots$ & $\cdots$ & 2.61 & 0.0089 & 0.0008 & -0.08 & 14.25 & M92 \\
\hline
\end{tabular}


Table 4-Continued

\begin{tabular}{|c|c|c|c|c|c|c|c|c|c|c|}
\hline LHS & V & B-V & V-R & R-I & V-I & $\pi\left({ }^{\prime \prime}\right)$ & $\sigma_{\pi}\left(^{\prime \prime}\right)$ & $\Delta_{L K}{ }^{\mathrm{a}}$ & $M_{V}$ & Source ${ }^{b}$ \\
\hline 3073 & 13.69 & 1.41 & 0.84 & 0.74 & 1.58 & 0.0187 & 0.0034 & -0.41 & 10.05 & Y,DF92 \\
\hline 3084 & 13.43 & 1.43 & 0.93 & 0.90 & 1.83 & 0.0190 & 0.0025 & -0.19 & 9.82 & Y,DF92 \\
\hline 3181 & 17.18 & 1.69 & 1.02 & 1.19 & 2.21 & 0.0265 & 0.0030 & -0.14 & 14.3 & RA \\
\hline 3189 & 18.10 & $\cdots$ & 1.11 & 1.70 & 2.81 & $\cdots$ & $\cdots$ & $\cdots$ & $\cdots$ & $\mathrm{HCM}$ \\
\hline 3192 & 14.76 & 1.63 & 1.11 & 1.38 & 2.49 & 0.0274 & 0.0023 & -0.07 & 11.95 & $\mathrm{Y}$, \\
\hline 3193 & 12.49 & 1.43 & 0.93 & 0.99 & 1.92 & 0.0274 & 0.0023 & -0.07 & 9.68 & Y,DF92 \\
\hline 3259 & 18.26 & $\ldots$ & $\cdots$ & $\cdots$ & 2.03 & 0.0064 & 0.0012 & -0.44 & 12.29 & M92 \\
\hline 3382 & 17.02 & 1.99 & 1.03 & 1.06 & 2.09 & 0.0104 & 0.0009 & -0.08 & 12.11 & M,L \\
\hline 3409 & 15.16 & 1.85 & 1.21 & 1.61 & 2.82 & 0.0500 & 0.0013 & -0.01 & 13.65 & Y,M92 \\
\hline 3480 & 17.24 & $\ldots$ & $\ldots$ & $\ldots$ & 2.76 & 0.0177 & 0.0008 & -0.02 & 13.48 & M92 \\
\hline 3481 & 17.58 & $\cdots$ & $\ldots$ & $\cdots$ & 2.35 & 0.0067 & 0.0007 & -0.12 & 11.71 & M92 \\
\hline 3548 & 17.52 & 1.78 & $\ldots$ & $\ldots$ & 2.09 & 0.0083 & 0.0006 & -0.05 & 12.12 & M,L \\
\hline 3555 & 17.93 & 1.94 & 1.10 & 0.97 & 2.07 & 0.0125 & 0.0060 & $\mathrm{~L}$ & 13.41 & $\mathrm{RA}$ \\
\hline 3628 & 17.41 & $\cdots$ & $\ldots$ & $\ldots$ & 2.05 & 0.0088 & 0.0008 & -0.09 & 12.13 & M92 \\
\hline 3684 & 17.95 & $\ldots$ & $\ldots$ & $\ldots$ & 2.92 & 0.0152 & 0.0010 & -0.04 & 13.86 & M92 \\
\hline 3867 & 13.41 & 1.19 & $\ldots$ & $\ldots$ & $\ldots$ & 0.0120 & 0.0050 & $\mathrm{~L}$ & 8.81 & $\mathrm{Y}, \mathrm{S}$ \\
\hline 3957 & 13.55 & 1.39 & $\ldots$ & $\ldots$ & $\ldots$ & 0.0257 & 0.0037 & -0.23 & 10.6 & $\mathrm{Y}, \mathrm{S}$ \\
\hline 4037 & 13.52 & 0.88 & $\ldots$ & 0.47 & $\ldots$ & $\cdots$ & $\cdots$ & $\cdots$ & $\cdots$ & R89 \\
\hline 6304 & 14.07 & $\ldots$ & 1.05 & 1.25 & 2.30 & $\ldots$ & $\ldots$ & $\ldots$ & $\ldots$ & W84 \\
\hline
\end{tabular}

${ }^{a} \mathrm{~L}$ indicates the Lutz-Kelker correction is undefined but $<-0.80$.

${ }^{\mathrm{b}}$ References for the trigonometric parallaxes and photometry are: B - Bessell (1990), CNS3 - Gliese and Jahreiss (1991), DF89 - Dawson and Forbes (1989), DF92 - Dawson and Forbes (1992), E79 - Eggen (1979), E87 - Eggen(1987), HD - Harrington and Dahn (1980), L - Leggett (1992), M92 - Monet et al. (1992), R - Ryan (1992), RA - Ruiz and Anguita (1993), S - SIMBAD, T - Tinney et al. (1995), W84 - Weis (1984), W88 - Weis (1987), W91 - Weis (1991), WU - Weis and Upgren (1982), Y - Van Altena et al. (1991)

${ }^{\mathrm{c}}$ Includes 0.75 magnitude correction for unresolved companion 
Table 5. Space Velocities

\begin{tabular}{|c|c|c|c|c|c|c|c|c|c|}
\hline LHS & $\mathrm{U}$ & $\mathrm{V}$ & W & $v_{t a n}$ & LHS & $\mathrm{U}$ & $\mathrm{V}$ & $\mathrm{W}$ & $v_{t a n}$ \\
\hline LHS 12 & -196 & -254 & 15 & 321 & LHS 453 & -239 & -148 & 371 & 465 \\
\hline LHS 20 & 140 & -185 & 76 & 221 & LHS 460 & 151 & -48 & 20 & 151 \\
\hline LHS 29 & 19 & -284 & -53 & 159 & LHS 467 & 282 & -90 & -117 & 260 \\
\hline LHS 42 & 178 & -416 & 180 & 465 & LHS 479 & 189 & -260 & -17 & 313 \\
\hline LHS 55 & -92 & -100 & 112 & 175 & LHS 482 & 103 & -116 & 19 & 114 \\
\hline LHS 57 & -148 & 0 & 16 & 89 & LHS 489 & 147 & -257 & -116 & 304 \\
\hline LHS 61 & 121 & -141 & -3 & 184 & LHS 491 & 245 & -210 & 70 & 298 \\
\hline LHS 64 & 259 & -190 & -88 & 237 & LHS 522 & 30 & -318 & -83 & 289 \\
\hline LHS 156 & 1 & -204 & -42 & 204 & LHS 522 & 30 & -318 & -83 & 289 \\
\hline LHS 161 & -84 & -236 & 94 & 265 & LHS 536 & -268 & -121 & -31 & 290 \\
\hline LHS 169 & 32 & -273 & 51 & 253 & LHS 1088 & -175 & -57 & 114 & 216 \\
\hline LHS 170 & 96 & -234 & -13 & 175 & LHS 1174 & -88 & -286 & -19 & 278 \\
\hline LHS 173 & 56 & -255 & 16 & 208 & LHS $1742 a$ & -229 & -263 & 108 & 293 \\
\hline LHS 174 & 139 & -382 & -127 & 362 & LHS 1970 & 158 & -318 & -49 & 359 \\
\hline LHS 178 & 60 & -165 & -6 & 165 & LHS 2110 & 154 & -103 & 4 & 181 \\
\hline LHS 182 & 123 & -335 & -118 & 291 & LHS 2497 & -71 & -38 & 21 & 76 \\
\hline LHS 185 & 4 & -289 & -169 & 333 & LHS 2715 & -57 & -94 & 28 & 108 \\
\hline LHS 192 & -143 & -463 & -60 & 482 & LHS 2852 & -34 & -5 & 57 & 65 \\
\hline LHS 205a & 119 & -455 & -45 & 466 & LHS 3061 & 73 & -277 & 103 & 297 \\
\hline LHS 211 & 200 & -247 & 99 & 307 & LHS 3073 & -239 & -238 & -76 & 234 \\
\hline LHS 216 & -321 & -311 & -14 & 216 & LHS 3084 & 31 & -223 & -65 & 230 \\
\hline LHS 218 & 4 & -188 & -77 & 202 & LHS 3192 & 45 & -136 & 72 & 160 \\
\hline LHS 236 & -207 & -229 & 93 & 315 & LHS 3193 & 55 & -126 & 85 & 160 \\
\hline LHS 276 & -115 & -115 & -47 & 163 & LHS 3259 & -189 & -382 & 125 & 388 \\
\hline LHS 301 & -7 & -179 & 8 & 153 & LHS 3382 & -302 & -232 & -60 & 360 \\
\hline LHS 307 & 173 & -247 & 33 & 296 & LHS 3409 & -30 & -100 & 42 & 79 \\
\hline LHS 320 & -38 & -215 & 0 & 203 & LHS 3480 & -13 & -72 & -124 & 144 \\
\hline LHS 343 & 107 & -418 & 89 & 408 & LHS 3481 & 274 & -314 & -6 & 410 \\
\hline LHS 364 & 120 & -53 & 19 & 132 & LHS 3548 & 335 & -331 & -67 & 470 \\
\hline LHS 375 & 24 & -188 & 155 & 166 & LHS 3628 & 273 & -215 & 112 & 365 \\
\hline LHS 376 & -173 & -295 & 98 & 356 & LHS 3684 & 162 & -197 & -58 & 251 \\
\hline LHS 377 & 207 & -125 & 125 & 205 & LHS 3867 & -149 & -186 & -78 & 227 \\
\hline LHS 407 & -215 & -160 & -24 & 206 & LHS 3957 & -148 & -125 & 40 & 179 \\
\hline LHS 410 & 165 & -190 & -112 & 276 & TVLM 832-42500 & -120 & -22 & 8 & 99 \\
\hline LHS 418 & 279 & -240 & -161 & 402 & & & & & \\
\hline
\end{tabular}


Table 6. Velocity Statistics

\begin{tabular}{rrrrrr}
\hline \hline \multicolumn{1}{c}{ Group } & $\mathrm{N}$ & $\langle V\rangle$ & $\sigma_{U}$ & $\sigma_{V}$ & $\sigma_{W}$ \\
\hline $\mathrm{dM}$ & 16 & -134 & 125 & 78 & 71 \\
$\mathrm{sdM}$ & 26 & -202 & 177 & 100 & 82 \\
$\mathrm{esdM}$ & 19 & -287 & 176 & 95 & 126 \\
$\mathrm{sdK}$ & 5 & -238 & 111 & 118 & 61 \\
strong CaH1 & 24 & -38 & 61 & 44 & 30 \\
weak CaH1 & 157 & -26 & 48 & 30 & 24 \\
Disk (RHG) & 514 & -22 & 43 & 31 & 25 \\
\hline
\end{tabular}




\section{REFERENCES}

Ake, T.B., \& Greenstein, J.L. 1980, ApJ, 240, 859

Allard, F., \& Hauschildt, P.H. 1995, ApJ, 445, 433

Baldwin, J.A., \& Stone, R.P.S. 1984, MNRAS, 206, 241

Baraffe, I., Chabrier, G., Allard, F., \& Hauschildt, P.H. 1995, ApJ, 446, L35

Baraffe, I., \& Chabrier, G. 1996, ApJ, 461, L51

Bessell, M.S. 1982, Proc. ASA, 4, 417

Bessell, M.S. 1990, A\&AS, 83, 357

Bessell, M.S. 1991, AJ, 101, 662

Boeshaar, P.C. 1976, Ph.D. Thesis, Ohio State University

Carney, B.W., Latham, D.W., \& Laird, J.B. 1988, AJ, 96, 560

Carney, B.W., Latham, D.W., \& Laird, J.B. 1988, AJ, 97, 423

Dahn, C.C., Liebert, J., Harris, H.C., \& Guetter, H.H. 1995, The Bottom of the Main Sequence And Beyond, edited by C.G. Tinney (Berlin, Springer), p.239

Dawson, P.C. 1986, ApJ, 311, 984

Dawson, P.C., \& De Robertis, M.M. 1988, AJ, 95, 1251

Dawson, P.C., \& De Robertis, M.M. 1988, AJ, 98, 1472

Dawson, P.C., \& Forbes, D. 1989, PASP, 101, 614

Dawson, P.C., \& Forbes, D. 1992, AJ, 103, 2063

Eggen, O.J. 1979, ApJS, 39, 89

Eggen, O.J. 1987, AJ, 92, 379

Eggen, O.J. 1996, AJ, 111, 466

Giclas, H.L., Burnham, R., \& Thomas, N.G. 1971, The Lowell Proper Motion Survey (Lowell Observatory, Flagstaff, Arizona)

Gizis, J.E., \& Reid, I.N. 1996, AJ, 111, 365

Gizis, J.E., Reid, I.N., Hawley, S.L. 1996, in preparation 
Gliese, W., \& Jahreiss, H. 1991, Preliminary Version of the Third Catalog of Nearby Stars (CNS3)

Greenstein, J.L. 1989, PASP, 101, 787

Gunn, J.E., \& Oke, J.B. 1983, ApJ, 266, 723

Hamilton, D., \& Stauffer, J.R. 1993, AJ, 105, 1855

Hanson, R.B. 1979, MNRAS, 186, 875

Harrington, R.S., \& Dahn, C.C. 1980, AJ, 85, 454

Harris, H.C., Baum, W.A., Hunter, D.A., \& Kreidl, T.J. 1991, AJ, 101, 677

Hartwick, F.D.A., Cowley, A.P., \& Mould, J.R. 1984, ApJ, 286, 269

Hawley, S.L., Gizis, J.E., \& Reid, I.N. 1996, A.J., in press

Henry, T.J., Kirkpatrick, J.D., \& Simons, D.A. 1994, AJ, 108, 1437

Holtzman, J.A., et al. 1995, PASP, 107, 1065

Jaschek, C., \& Jaschek, M. 1987, The Classification of Stars (Cambridge University Press, Cambridge)

Jones, H.R.A., Longmore, A.J., Allard, F., \& Hauschildt, P.H. 1996, MNRAS, 280, 77

Joy. 1947, ApJ, 105, 96

Kirkpatrick, D.W., Henry, T.J., McCarthy, D.W. 1991, ApJS, 77, 417

Kuijken, K., \& Gilmore, G. 1989, MNRAS, 239, 605

Laird, J.B., Rupen, M.P., Carney, B.W., Latham, D.W. 1988, AJ, 96, 1908

Leggett, S.K. 1992, ApJS, 82, 351

Leggett, S.K., Allard, F., Berriman, G., Dahn, C.C., \& Hauschildt, P.H. 1996, ApJS, 104, 117

Liebert, J., Dahn, C.C., Gresham, M., Strittmatter, P.A. 1979, ApJ, 233, 226

Lutz, T.E., \& Kelker, D.H. 1973, PASP, 85, 573

Luyten, W.J. 1979, Catalogue of stars with proper motions exceeding 0".5 annually (LHS), (University of Minnesota, Minneapolis, Minnesota)

Majewski, S.R. 1993, ARA\&A31, 575

Marcy, G.W., \& Benitz, K.J. 1989, ApJ, 344, 441 
McCarthy, J.K. 1985, Proc. SPIE, 554, 155

Metcalfe, T.S., Mathieu, R.D., Latham, D.W., \& Torres, G. 1996, ApJ, 456, 356

Mihalas, D.M. \& Binney, J. 1981, Galactic Astronomy: Structure and Kinematics, 2nd ed. (W.H. Freeman, San Francisco)

Monet, D.G., Dahn, C.C., Vrba, F.J., Harris, H.C., Pier, J.R., Luginbuhl, C.B., \& Ables, H.D. 1992, AJ, 103, 638

Morgan, W.W., Keenan, P.C., \& Kellman, E. 1943, An Atlas of Stellar Spectra (Chicago: Univ. of Chicago Press)

Mould, J.R. 1976, A\&A, 48, 443

Mould, J.R., \& McElroy, D.B. 1978, ApJ, 220, 935

Norris, J. 1986, ApJS, 61, 667

Oort, J.H. 1965, Stars and Stellar Systems V, Galactic Structure, edited by A. Blaauw \& M. Schmidt (University of Chicago Press), p. 500.

Reid, I.N. 1982, MNRAS, 201, 51

Reid, I.N., Hawley, S.L., \& Gizis, J.E. 1995, AJ, 110, 1838

Reid, I.N., Yan, L., Majewski, S., Thompson, I., \& Smail, I.R. 1996, A.J., in press

Richer, H.B., \& Fahlman, G.G. 1992, Nature, 358, 383

Ruiz, M.T., \& Anguita, C. 1993, AJ, 105, 614

Ryan, S.G. 1992, AJ, 104, 1144

Santiago, B.X., Elson, R.A.W., \& Gilmore, G.F. 1996, MNRAS, 281, 1363

Schmidt, M. 1975, ApJ, 202, 22

Tinney, C.G., Reid, I.N., Gizis, J., \& Mould, J.R. 1995, AJ, 110, 3014

Van Altena, W.F., Lee, J.T., \& Hoffleit, E.D. 1991, The General Catalog of Trigonometric Stellar Parallaxes: A Preliminary Version

Weis, E.W. 1984, ApJS, 55, 289

Weis, E.W. 1988, AJ, 96, 171

Weis, E.W. 1991, AJ, 101, 1882

Weis, E.W., \& Upgren, A.R. 1982, PASP, 94, 821 
Weistrop, D. 1977, ApJ, 215, 845

Young, A., Sadjai, S., \& Harlan, E. 1987, ApJ, 314, 272

This preprint was prepared with the AAS $\mathrm{IAT}_{\mathrm{E}} \mathrm{X}$ macros v4.0. 


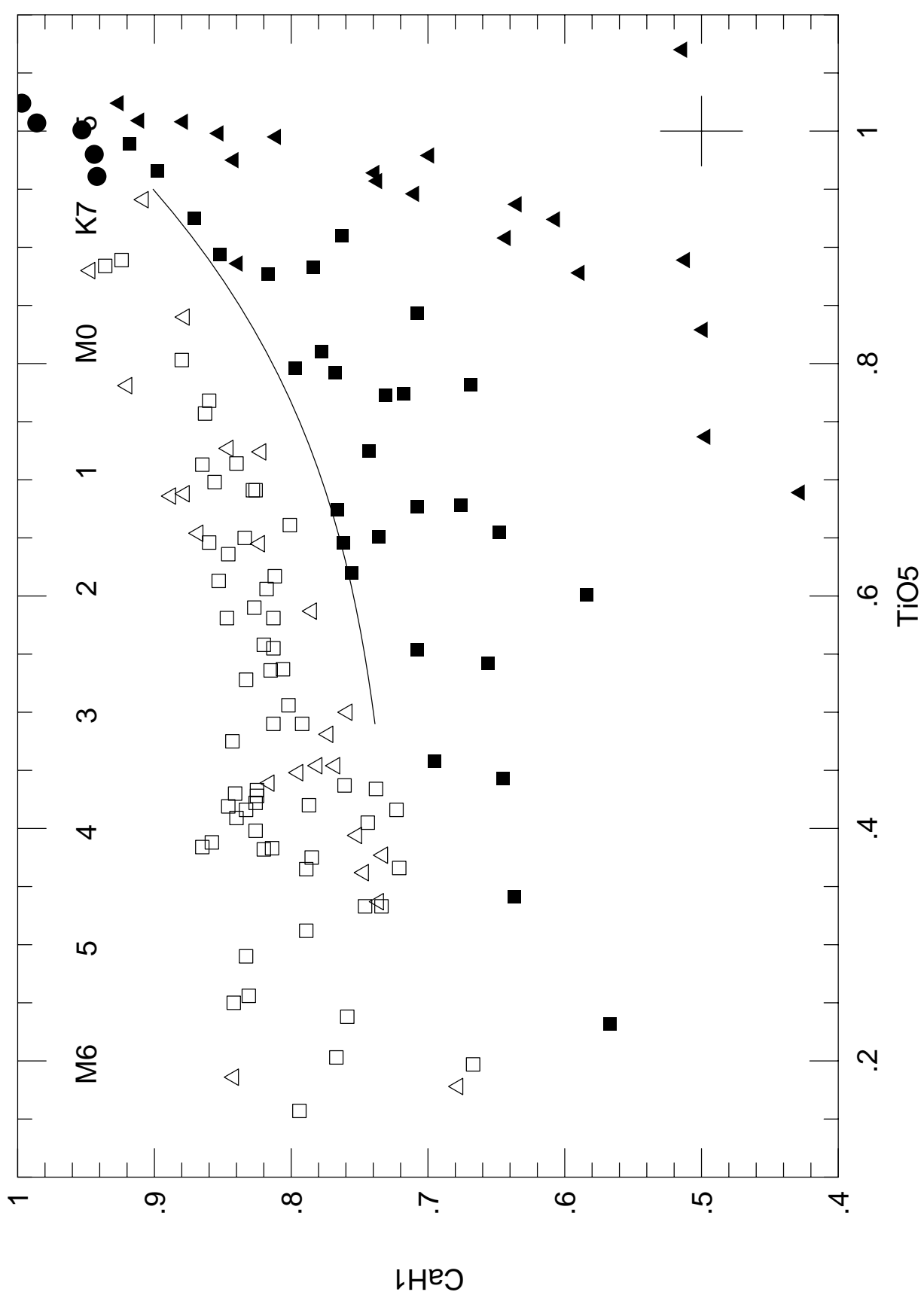

Fig. 1. - The CaH indices as a function of TiO5. Stars classified as sdM appear as filled squares, esdM as filled triangles, sdK as filled circles, and spectroscopic non-subdwarfs as open triangles. In the CaH1 diagram we also plot the single stars within eight parsecs as open squares. All other figures use the same symbols. Representative error bars $( \pm 0.03)$ appear in the lower right. 


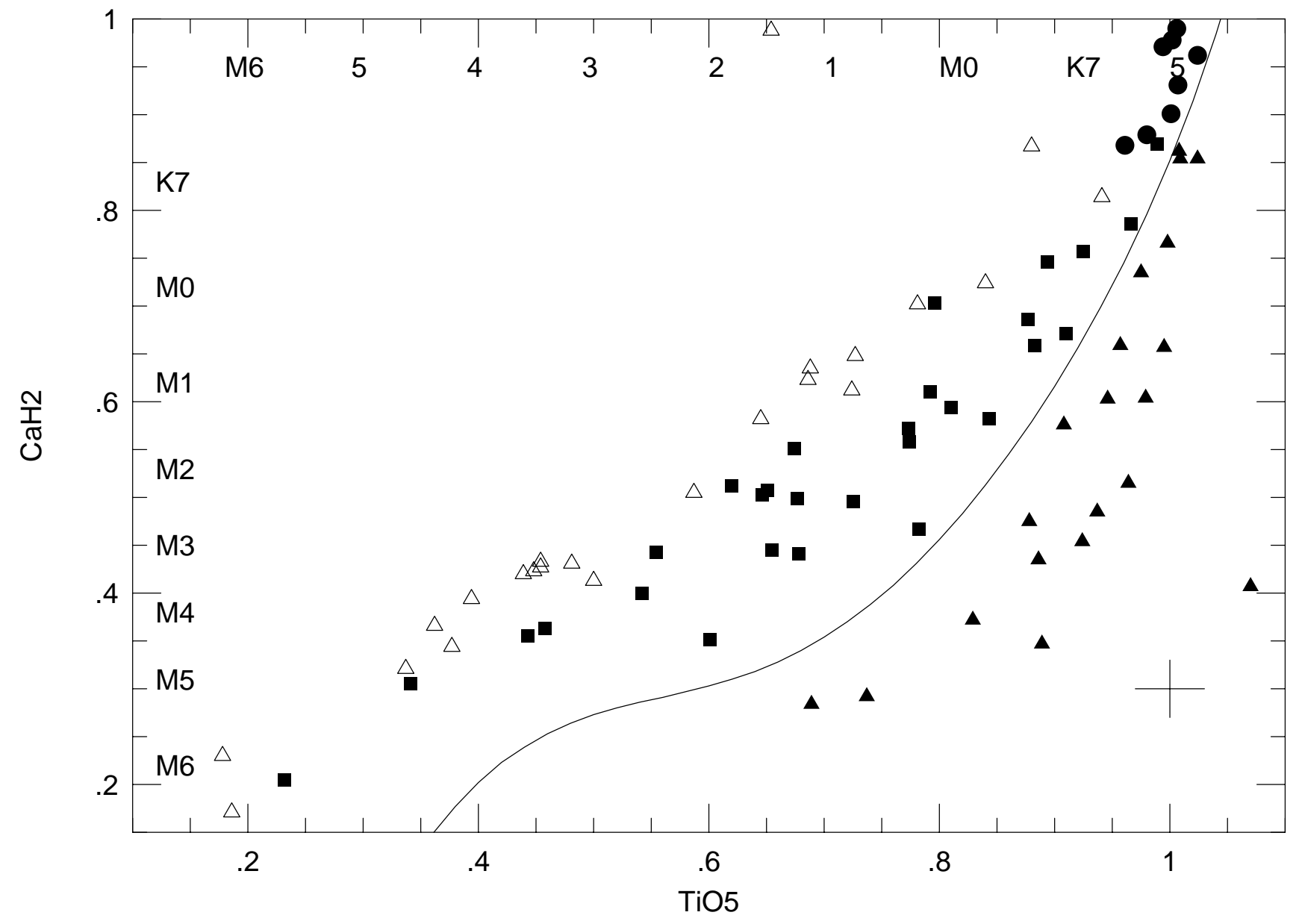




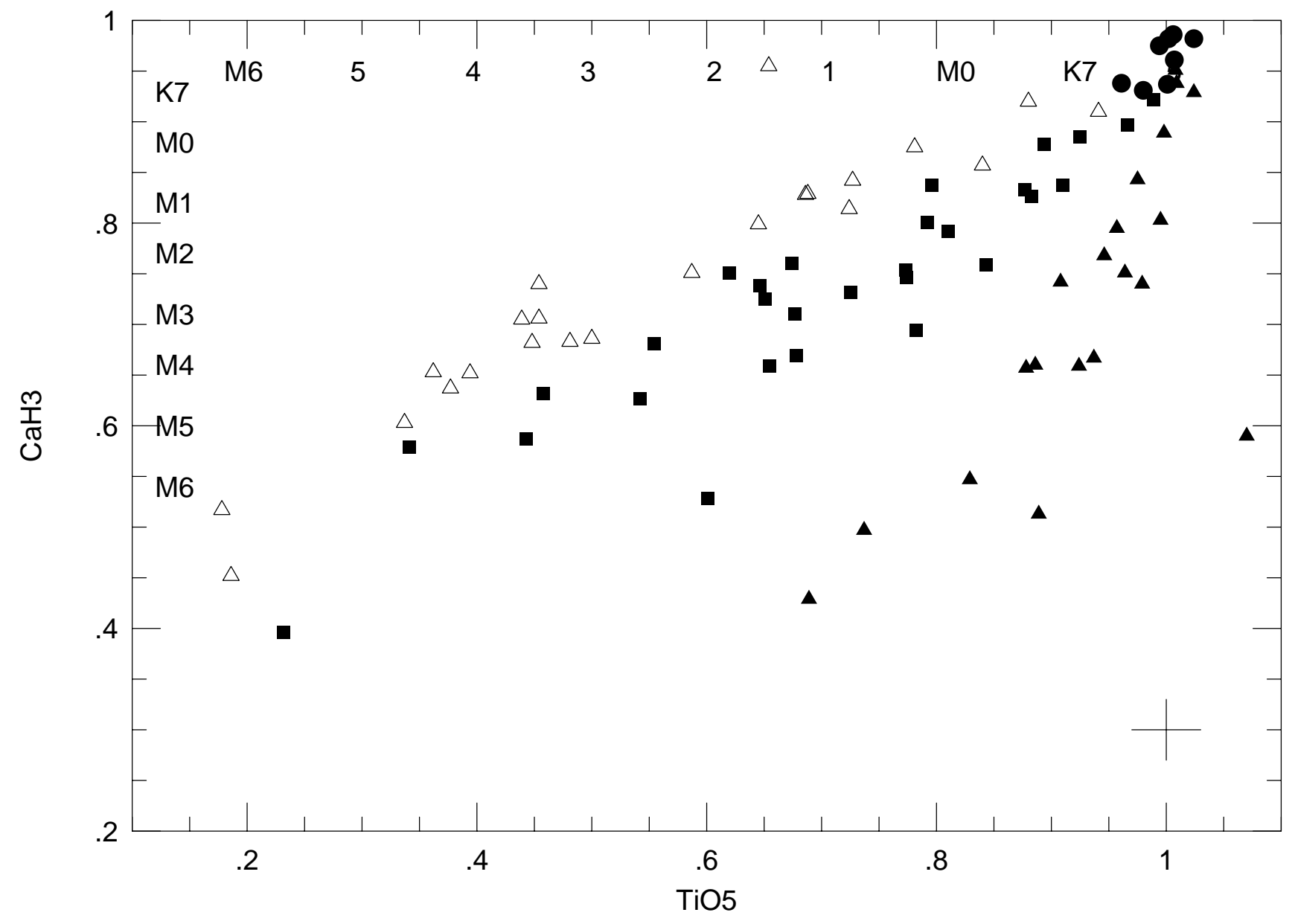



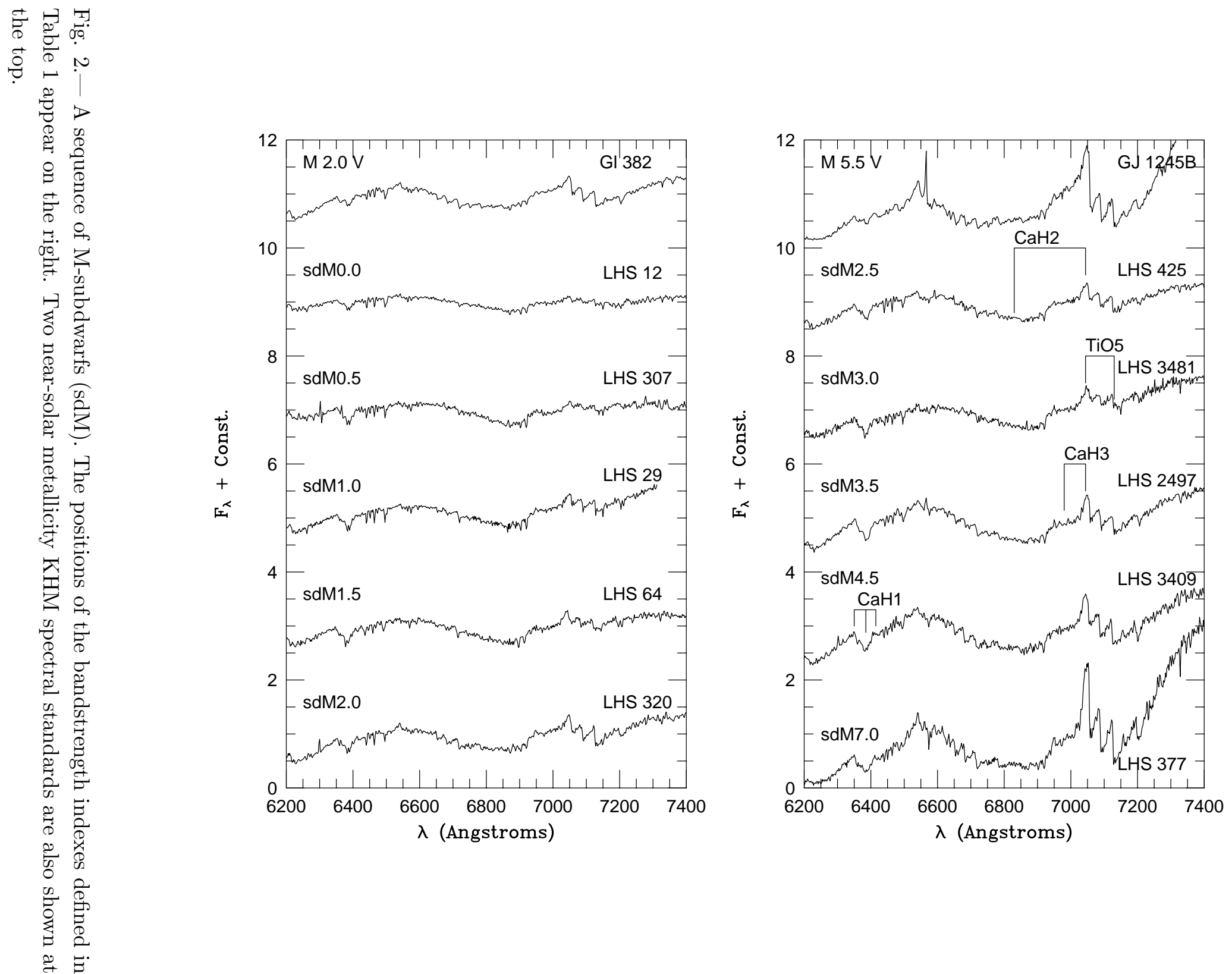

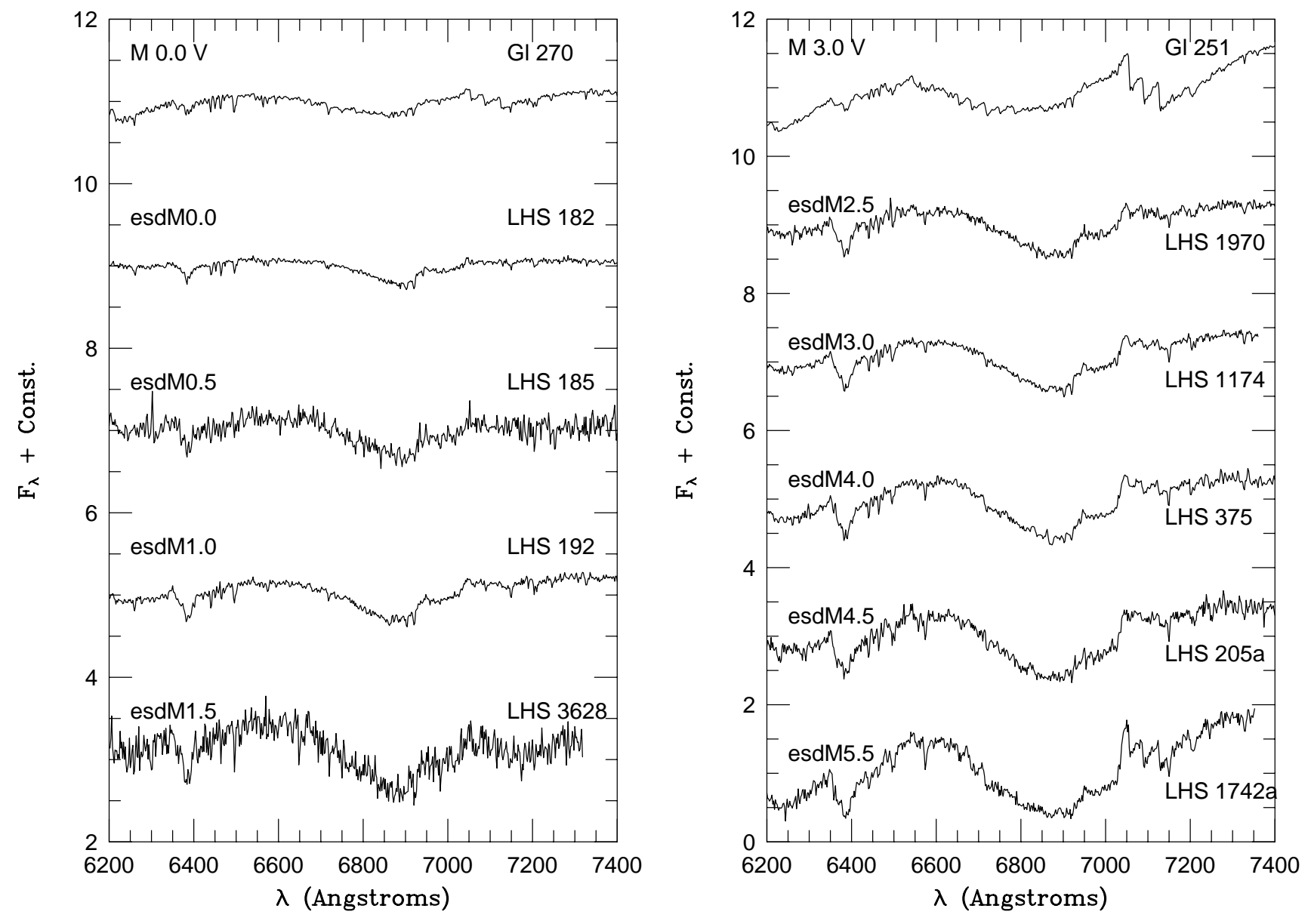


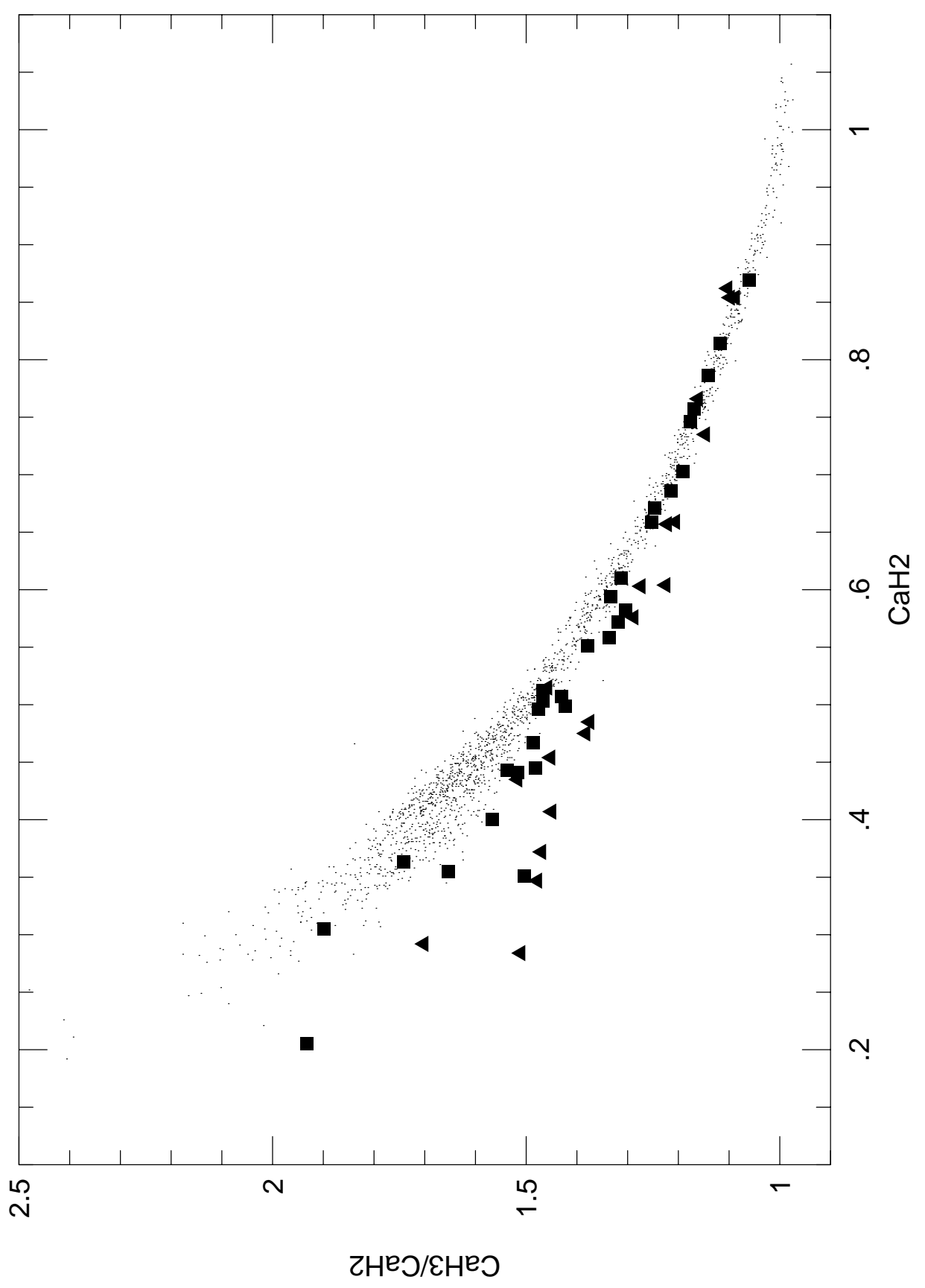

Fig. 4. - The CaH3/CaH2 ratio as function of $\mathrm{CaH} 2$. The sdM and esdM lie below the RHG Population I relation. The RHG stars appear as dots. 


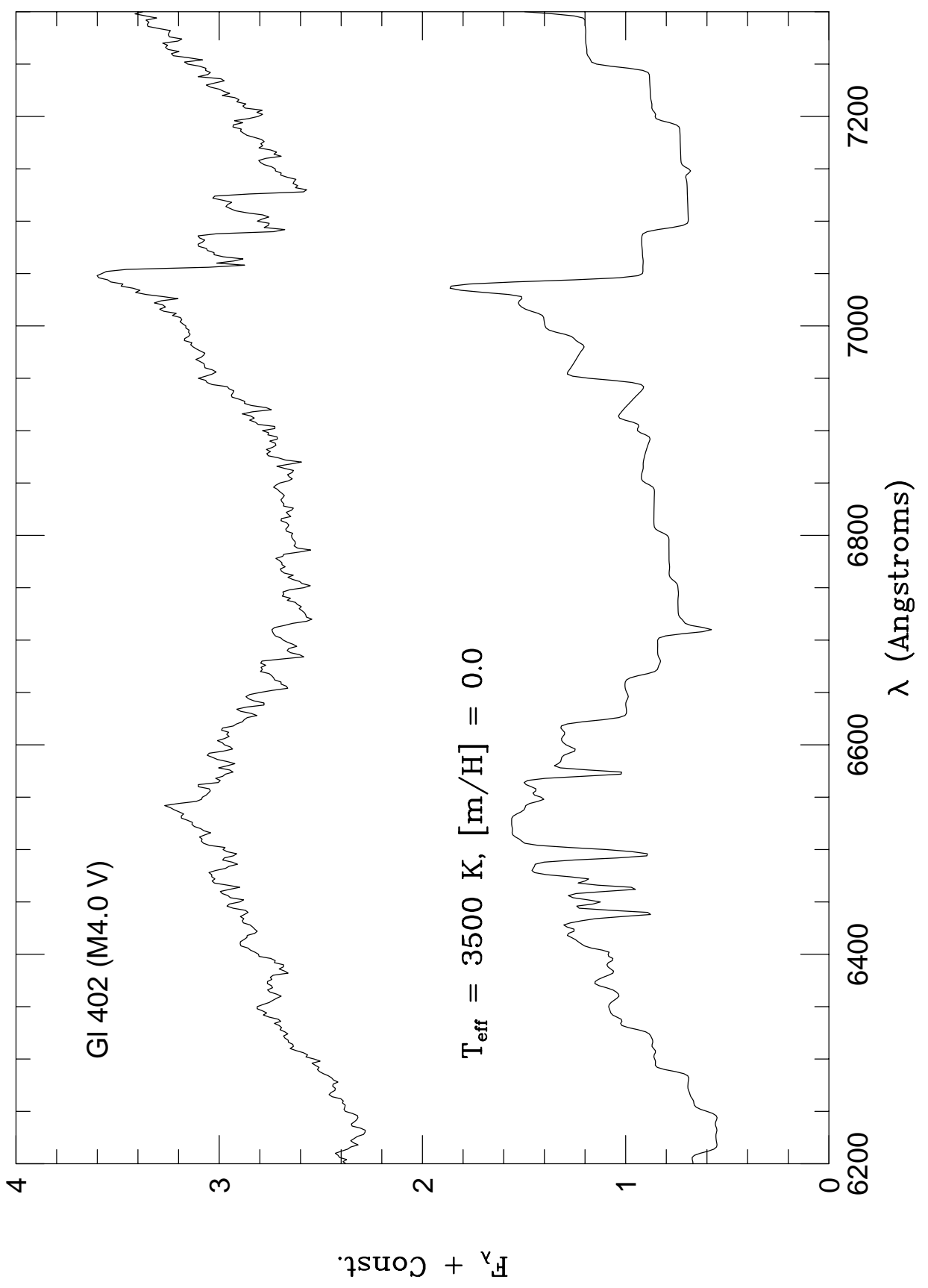

Fig. 5.- A model atmosphere fit to the Population I star Gl 402, an M $4.0 \mathrm{~V}$ standard. Note that the overly strong atomic lines are an artifact of the computation technique. 


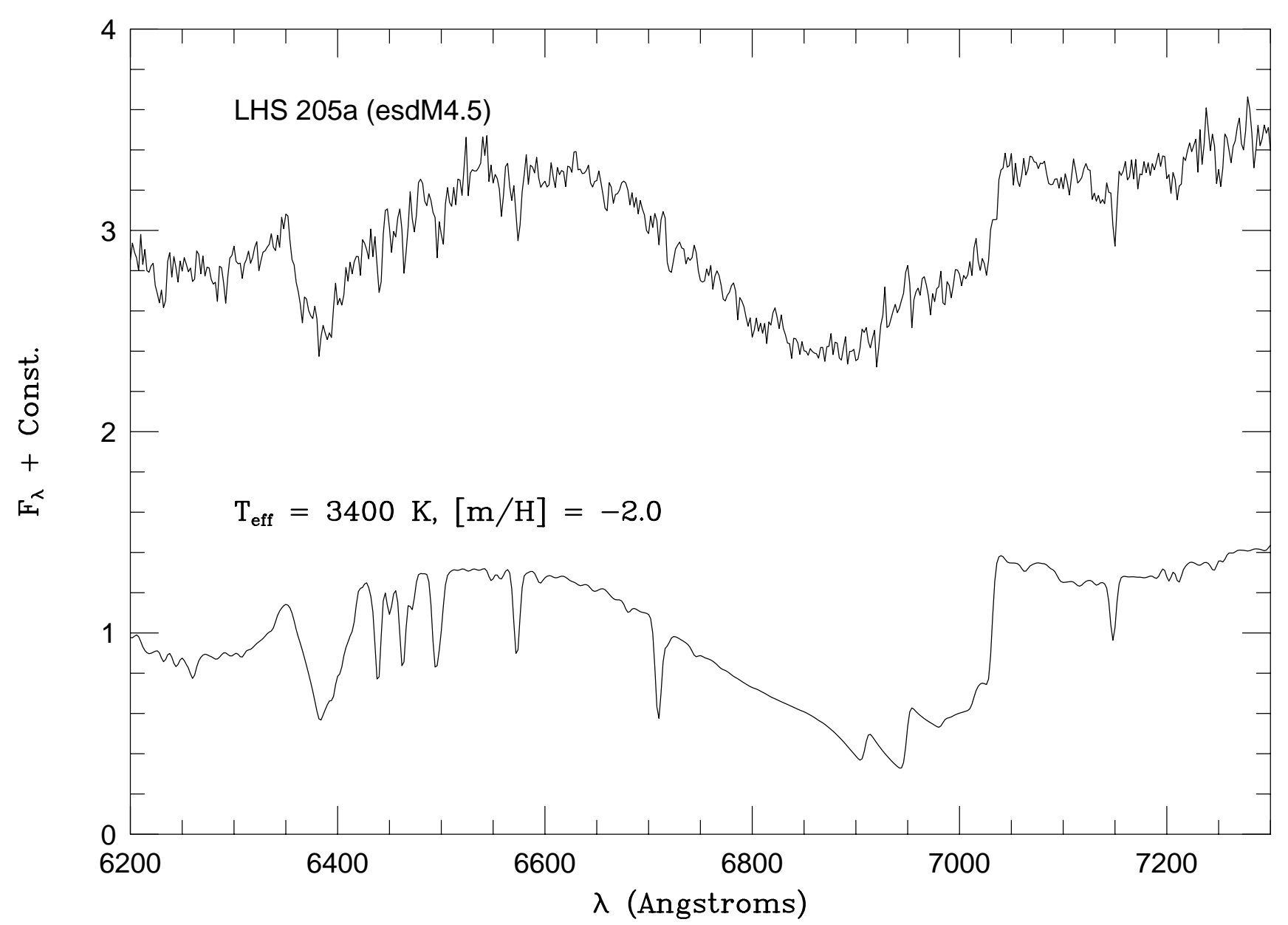




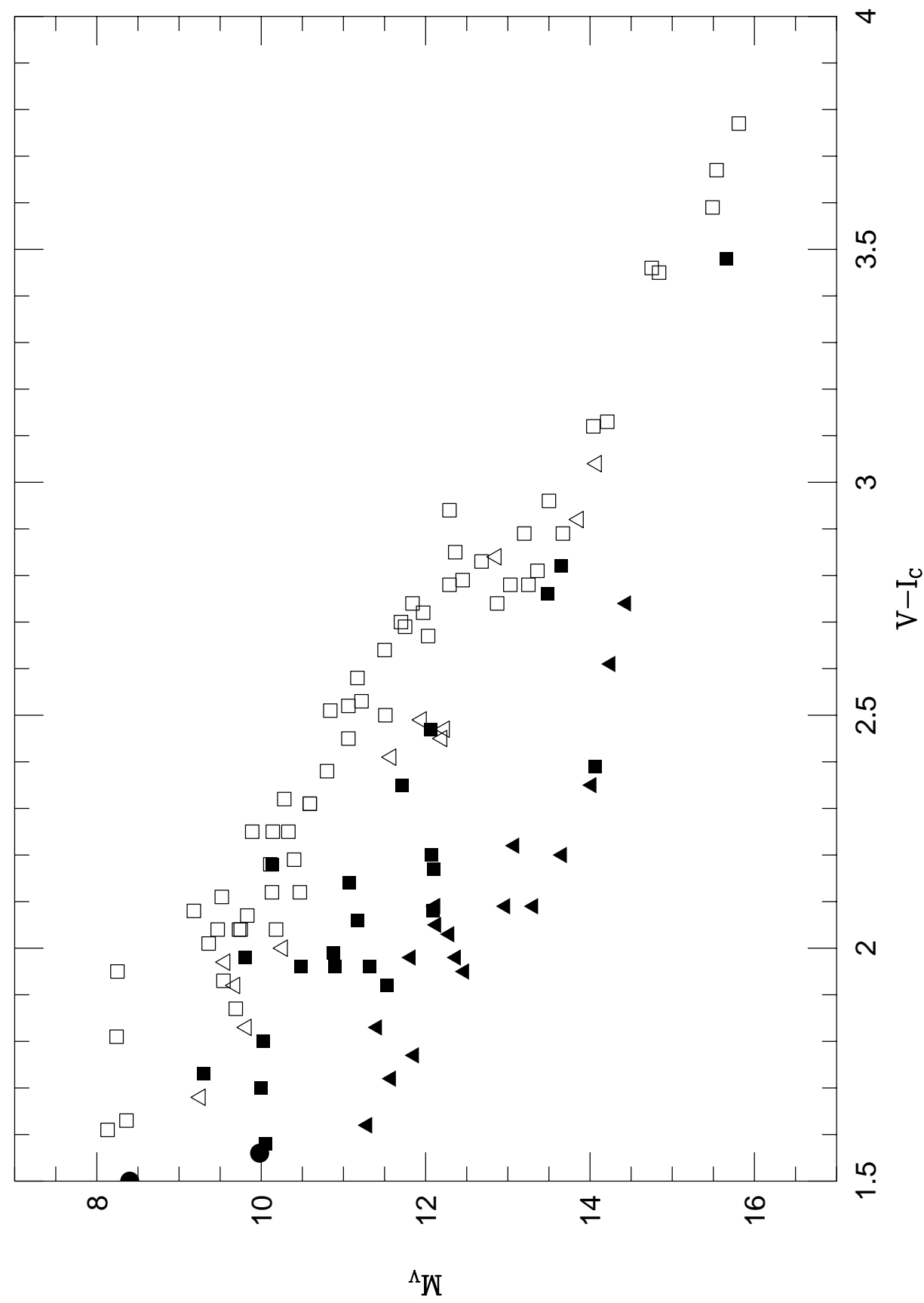

Fig. 7.- The observed HR diagram. The spectroscopic extreme subdwarfs (esdM) are filled triangles, the intermediate subdwarfs (sdM) are filled squares, and the subdwarf candidates that were spectroscopically indistinguishable are open triangles. The open squares are the single stars within eight parsecs with good parallaxes - note the step at V-I $\sim 2.8$ which is discussed in HGR. 


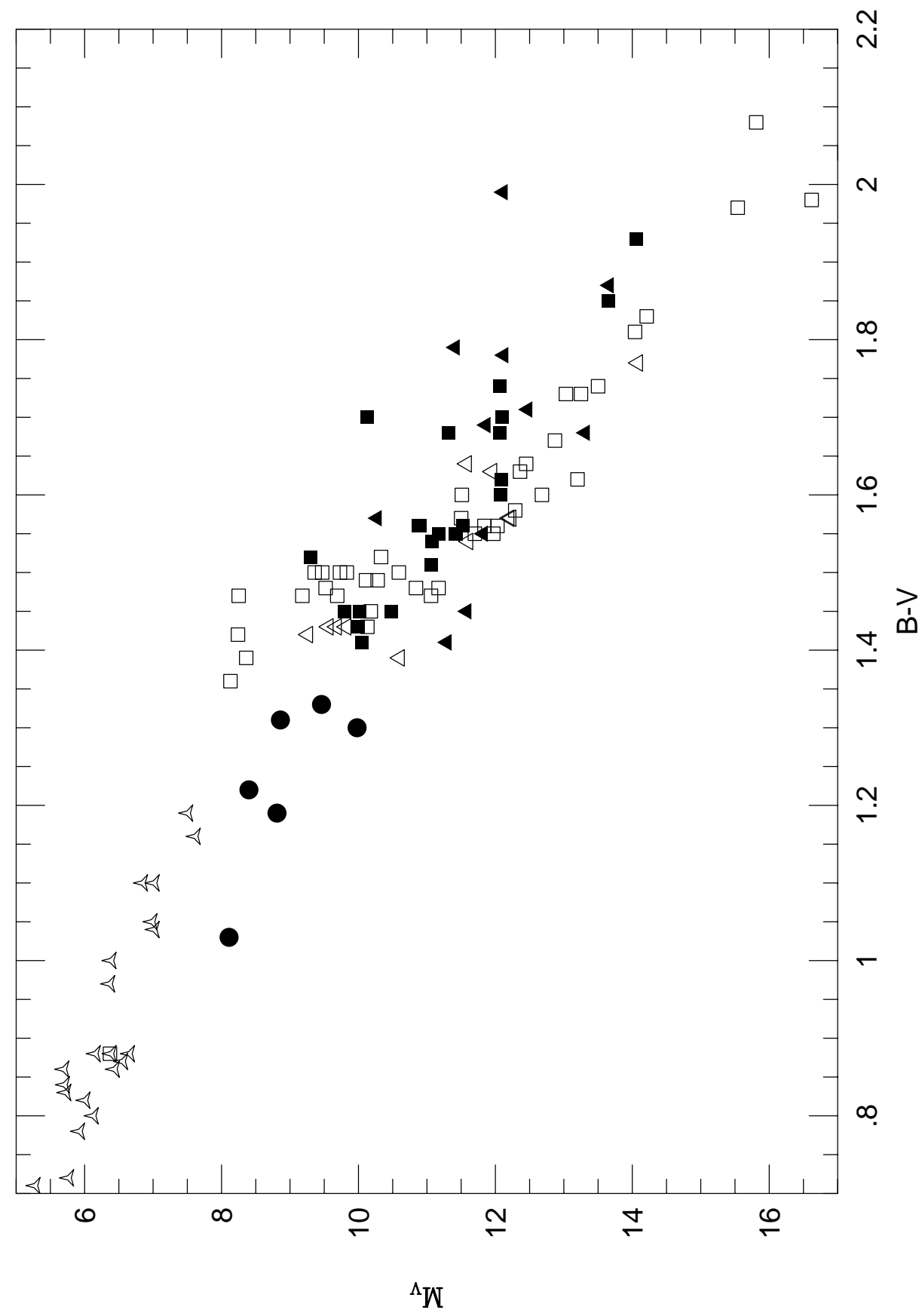

Fig. 8. - The HR diagram for B-V colors. The three pointed stars are nearby $\mathrm{G}$ and $\mathrm{K}$ dwarfs. Note that the blue K-subdwarfs lie below the disk sequence, but the sdM and esdM sequences cross the disk main sequence and are actually brighter at the reddest colors. 


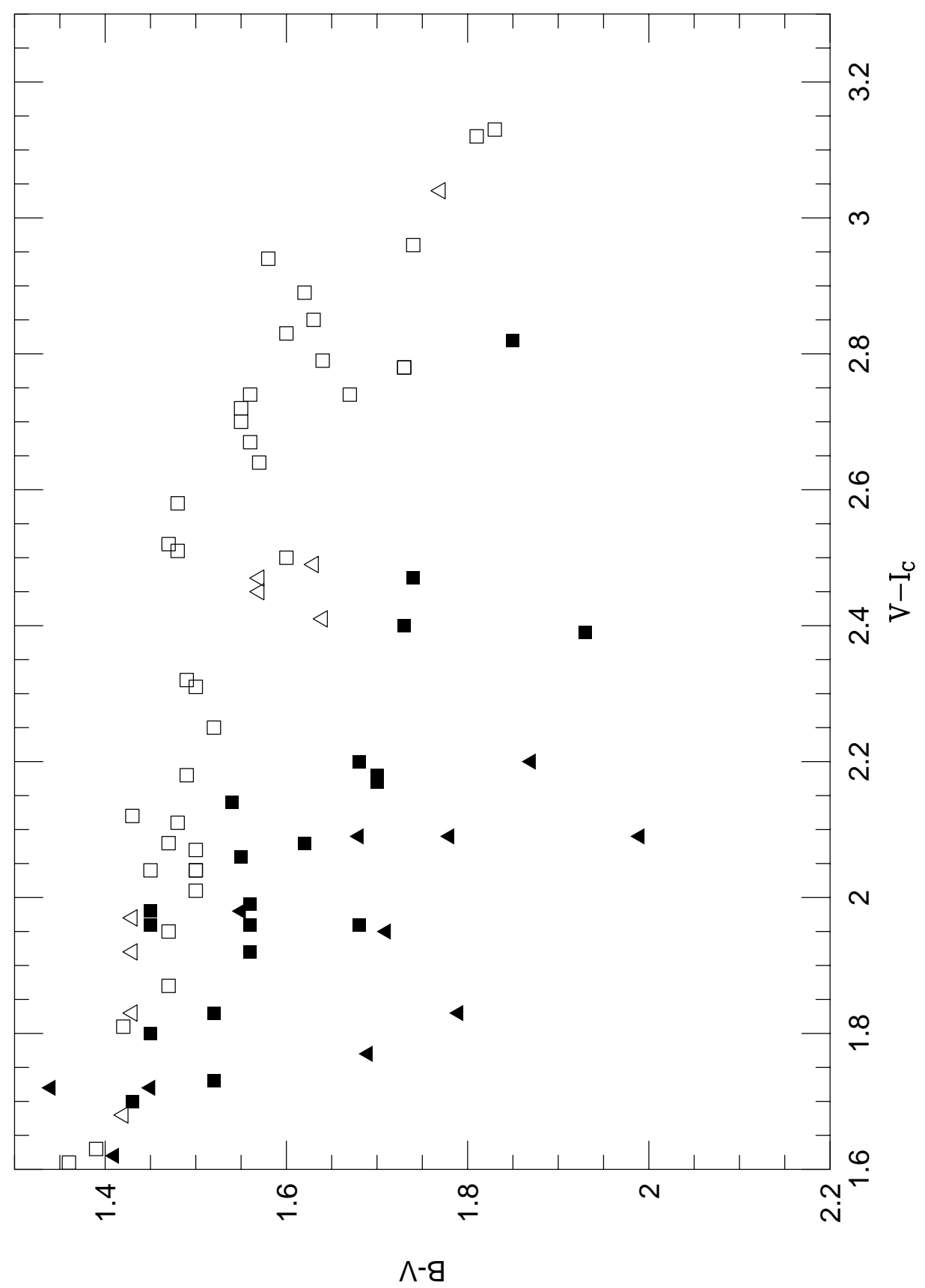

Fig. 9.- V-I vs. B-V. The sdM are redder in B-V at a given V-I color than the disk stars. The esdM show a larger offset than the sdM 


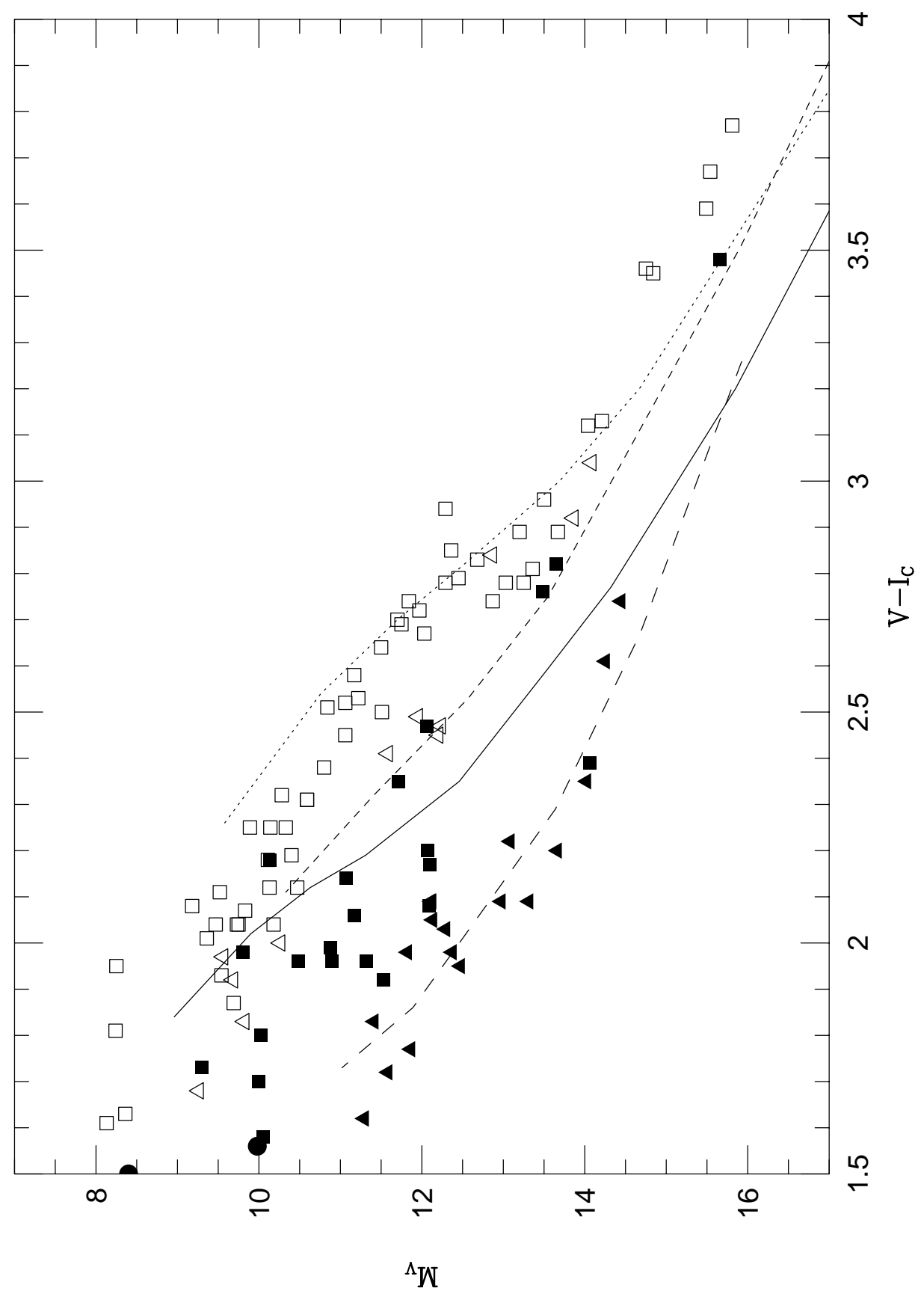

Fig. 10. - The observed HR diagram with theoretical sequences. Metallicities of $[m / H]=0.0$ (dotted line ), -0.5 (short dashed line), and -1.5 (long dashed line) from Baraffe et al. (1995). A more recent solar metallicity model (solid line) computed by Baraffe and Chabrier using the NextGen model atmospheres is also shown. The latter model does not match the observed disk stars, but the agreement of the previous models is quite good. 


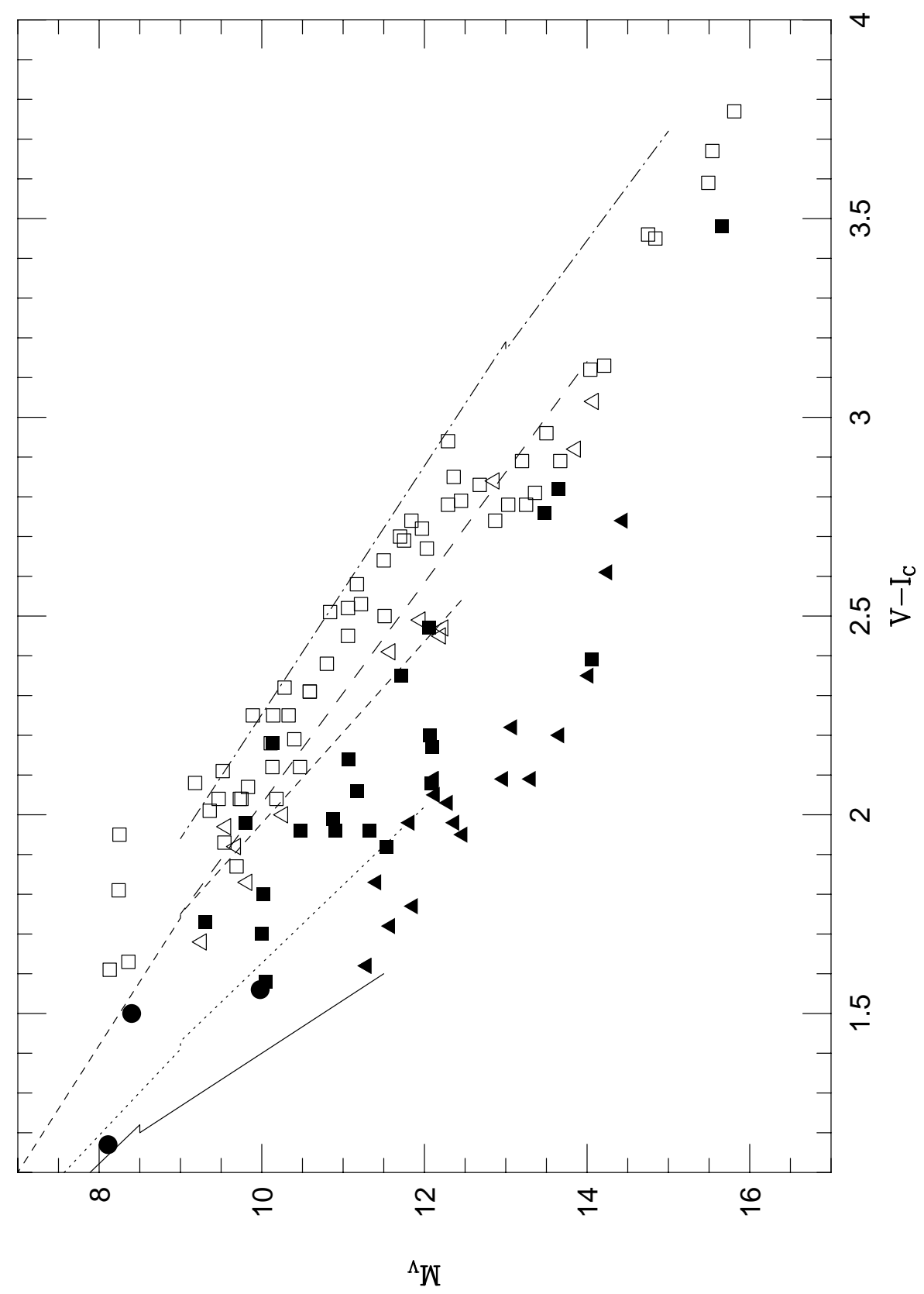

Fig. 11. - The observed HR diagram with the cluster main sequences fit by Santiago et al.(1996) using HST observations. From left to right, the clusters are M15 (solid line, $[\mathrm{Fe} / \mathrm{H}]=-2.26$ ), $\omega$ Cen (dotted, -1.6), 47 Tuc (short dashed, -0.6), N2420 (long dashed, -0.45), and N 2477 (long dash-dotted, 0.00). Note that the position of the cluster sequences agrees with our spectroscopic metallicity estimates. The slopes of the cluster sequences do not disagree with the local subdwarfs in the region of color overlap when using our classifications. The discrepancy of the reddest stars in the metal rich clusters may be due to color terms, as well as the inadequacy of a linear fit for the main sequence. 